\title{
Computational Nuclear Quantum Many-Body Problem: The UNEDF Project
}

S. Bogner ${ }^{\mathrm{i}}$, A. Bulgac ${ }^{\mathrm{x}}$, J. Carlson ${ }^{\mathrm{f}}$, J. Engel ${ }^{\mathrm{t}}$, G. Fann ${ }^{\mathrm{j}}$, R.J. Furnstahl ${ }^{\mathrm{m}}$, S. Gandolfi ${ }^{\mathrm{f}}$, G. Hagen ${ }^{\mathrm{l}}$, M. Horoi $^{\mathrm{d}}$, C. Johnson ${ }^{\mathrm{q}}$, M. Kortelainen ${ }^{\mathrm{u}, \mathrm{l}, \mathrm{s}}$, E. Lusk ${ }^{\mathrm{a}}$, P. Maris ${ }^{\mathrm{e}}$, H. Nam ${ }^{\mathrm{k}}$, P. Navratil ${ }^{\mathrm{h}, \mathrm{r}}$, W. Nazarewicz ${ }^{\mathrm{u}, 1, \mathrm{w}}$, E. Ng ${ }^{\mathrm{g}}$, G.P.A. Nobre ${ }^{\mathrm{c}}$, E. Ormand ${ }^{\mathrm{h}}$, T. Papenbrock ${ }^{\mathrm{l}, \mathrm{u}}$, J. Pei ${ }^{\mathrm{u}, \mathrm{l}, \mathrm{p}}$, S. C. Pieper ${ }^{\mathrm{b}}$, S. Quaglioni ${ }^{\mathrm{h}}$, K.J. Roche ${ }^{\mathrm{o}, \mathrm{x}}$, J. Sarich ${ }^{\mathrm{a}}$, N. Schunck ${ }^{\mathrm{h}}$, M. Sosonkina ${ }^{\mathrm{n}}$, J. Terasaki ${ }^{\mathrm{v}, \mathrm{t}}$, I. Thompson ${ }^{\mathrm{h}}$, J.P. Vary ${ }^{\mathrm{e}}$, S.M. Wild ${ }^{\mathrm{a}}$

${ }^{a}$ Mathematics and Computer Science Division, Argonne National Laboratory, Argonne, IL 60439, USA

${ }^{b}$ Physics Division, Argonne National Laboratory, Argonne, IL 60439, USA

${ }^{c}$ National Nuclear Data Center, Brookhaven National Laboratory, Upton, NY 11973, USA

${ }^{d}$ Central Michigan University, Mount Pleasant, MI 48859, USA

${ }^{e}$ Department of Physics and Astronomy, Iowa State University, Ames, IA 50011, USA

${ }^{f}$ Theoretical Division, Los Alamos National Laboratory, Los Alamos, NM 87545, USA

${ }^{g}$ Computational Research Division, Lawrence Berkeley National Laboratory, Berkeley, CA 94720, USA

${ }^{h}$ Physics Division, Lawrence Livermore National Laboratory, Livermore, CA 94551, USA

${ }^{i}$ National Superconducting Cyclotron Lab, Michigan State University, East Lansing, MI, 48824, USA

${ }^{j}$ Computer Science and Mathematics Division, Oak Ridge National Laboratory, Oak Ridge, TN 37831, USA

${ }^{k}$ National Center for Computational Sciences Division, Oak Ridge National Laboratory, Oak Ridge, TN 37831 , USA

${ }^{l}$ Physics Division, Oak Ridge National Laboratory, Oak Ridge, TN 37831, USA

${ }^{m}$ Department of Physics, Ohio State University, Columbus, OH 43210, USA

${ }^{n}$ Department of Modeling, Simulation and Visualization Engineering, Old Dominion University, Norfolk, VA 23529, USA

${ }^{\circ}$ Computational Sciences and Mathematics Division, Pacific Northwest National Laboratory, Richland, WA 99352, USA

${ }^{p}$ State Key Laboratory of Nuclear Physics and Technology, School of Physics, Peking University, Beijing 100871, China

${ }^{q}$ Department of Physics, San Diego State University, San Diego, CA 92182, USA

rTRIUMF, 4004 Westbrook Mall, Vancouver, BC, V6T 2A3, Canada

${ }^{s}$ Department of Physics, P.O. Box 35 (YFL), FI-40014, University of Jyväskylä, Finland

${ }^{t}$ Department of Physics and Astronomy, University of North Carolina, Chapel Hill, NC 27599, USA

${ }^{u}$ Department of Physics and Astronomy, University of Tennessee, Knoxville, TN 37996, USA

${ }^{v}$ Division of Physics and Center for Computational Sciences, University of Tsukuba, Tsukuba, 305-8577, Japan

${ }^{w}$ Faculty of Physics, University of Warsaw, 00-681 Warsaw, Poland

${ }^{x}$ Department of Physics, University of Washington, Seattle, WA 98195, USA

\section{Abstract}

The UNEDF project was a large-scale collaborative effort that applied high-performance computing to the nuclear quantum many-body problem. UNEDF demonstrated that close associations among nuclear physicists, mathematicians, and computer scientists can lead to novel physics outcomes built on algorithmic innovations and computational developments. This review showcases a wide range of UNEDF science results to illustrate this interplay.

Keywords: Configuration interaction, Coupled-cluster method, Density functional theory, Effective field theory, High-performance computing, Quantum Monte Carlo

\section{Introduction to UNEDF}

Understanding the properties of atomic nuclei is crucial for a complete nuclear theory, for element formation, for properties of stars, and for present and future energy and defense applications. From 2006 to 2012, the UNEDF (Universal Nuclear Energy Density Functional) collaboration carried out a comprehensive study of the nuclear many-body problem using advanced numerical algorithms and extensive computational resources, with a view toward scaling to petaflop supercomputing platforms and beyond.

The UNEDF project was carried out as part of the SciDAC (Scientific Discovery through Advanced Computing) program led by Advanced Scientific Computing Research (ASCR), part of the Office of Science in the U.S. Department of Energy (DOE). The SciDAC program was started in 2001 as a way to couple the applied mathematics and computer science research sponsored by ASCR to applied computational science application projects traditionally supported by other offices in DOE. UNEDF was funded jointly by ASCR, the Nuclear Physics program of the Office of Science, and the National Nuclear Security Administration. Over 50 physicists, applied mathematicians, and computer scientists from 9 universities and 7 national laboratories in the United States, as well as many international collaborators, participated in UNEDF.

This review describes science outcomes in nuclear many-body physics, with an emphasis on computational and algorithmic developments, that have resulted from the successful collaborations within UNEDF among mathematicians and computer scientists on one side and nuclear physicists on the other. Such collaborations "across the di- 
vide" were newly formed at the early stage of the project and became its unique feature, with high-performance computing serving as a catalyst for new interactions. The results described in this paper could not have been achieved without such couplings.

\subsection{UNEDF science}

The long-term vision initiated with UNEDF is to arrive at a comprehensive, quantitative, and unified description of nuclei and their reactions that is grounded in the fundamental interactions between the constituent nucleons [1, 2]. The goal is to replace phenomenological models of nuclear structure and reactions with a well-founded microscopic theory that delivers maximum predictive power with well-quantified uncertainties. Specifically, the mission of UNEDF was threefold:

1. Find an optimized energy density functional (EDF) using all our knowledge of the nucleonic Hamiltonian and basic nuclear properties.

2. Validate the functional using the relevant nuclear data.

3. Apply the validated theory to properties of interest that cannot be measured.

The main physics areas of UNEDF, defined at the beginning of the project [1, were ab initio structure, ab initio functionals, density functional theory (DFT) applications, DFT extensions, and reactions. Few connections between these areas existed at that time. As UNEDF matured, however, coherence grew within the effort. Indeed, the project created and facilitated an increasing interplay among the major areas where none had existed previously. Each of the main physics areas now includes ongoing collaborations that cross over into other areas. These interconnections are highlighted in the summary diagram of the UNEDF strategy shown in Fig. 1. In addition to physics links, numerous computer science/applied mathematics (CS/AM) interconnections were established within UNEDF as computational and mathematical tools developed in one area of UNEDF were used in other parts of the project. These tools, motivated by nuclear needs, are now available for other areas of science. Access to leadershipclass computing resources and large-scale compute time allocations were critical for the scientific investigations.

At the intersection of the ab initio techniques and DFT techniques are comparisons of observables among the various approaches, particularly through constraints on density. Such calculations have not been performed before and require significant computational capability and an increasing sophistication of data manipulation. Research on the nuclear problem would be incomplete without a serious effort to understand the nuclear interactions involved and their connection to DFT. Therefore, the UNEDF project also included elements that required less computational capability but are integral to the project, such as the development of nuclear forces using renormalization group

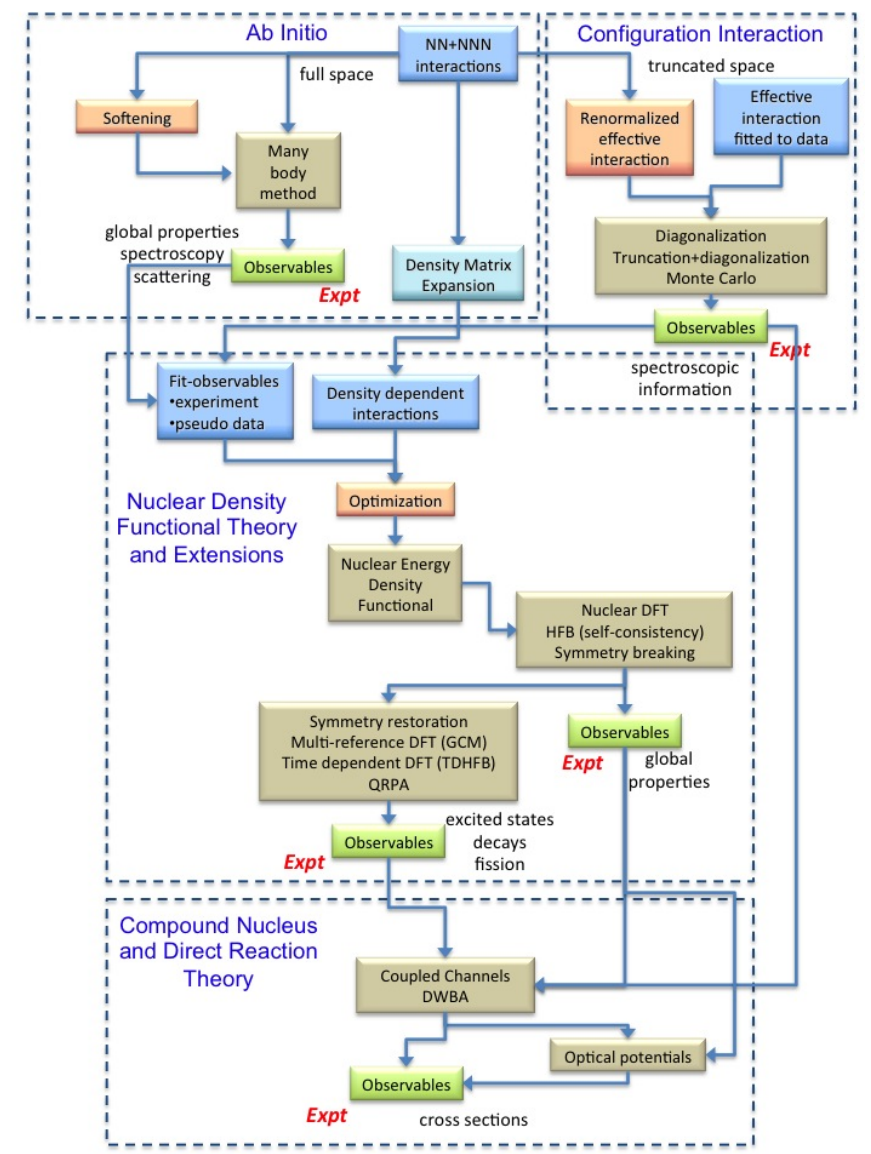

Figure 1: UNEDF project scope. Major science areas are indicated by boxes; interconnections between areas are marked by arrows. The green boxes indicate connections to experimental observations.

approaches. Another example is research on nuclear reaction properties that requires both the use and development of algorithms for the largest computers and more conventional computing needed for algorithmic breakthroughs.

Another new aspect of the nuclear theory effort driven by this project is a greatly enhanced degree of quality control. Integral to UNEDF was the verification of methods and codes, the estimation of uncertainties, and other output assessments. Methods used for verification and validation included the crosschecking of different theoretical methods and codes, the use of multiple DFT solvers with benchmarking, and benchmarking of different ab initio methods using the same Hamiltonian. A new way to estimate theory error bars was to use multiple Hamiltonians with different energy/momentum cutoffs and then analyze the cutoff dependence of calculated observables. The UNEDF assessment component necessitated the development and application of statistical tools to deliver uncertainty quantification and error analysis for theoretical studies as well as to assess the significance of new experimental data. Such technologies are essential as new theories and computational tools are applied to entirely new nuclear systems and to conditions that are not accessible to experiment. 


\subsection{Collaborative effort}

The successes of the UNEDF project were built upon certain best practices, some implemented originally and some learned by experience, in organizing and implementing the scientific effort. In order to foster the close alignment of the necessary applied mathematics and computer science research with the necessary physics research, multiple direct partnerships were formed consisting of computer scientists and applied mathematicians linked with specific physicists to remove algorithmic and/or computational barriers to progress. The five-year lifetime of the project provided time for these collaborations to become deep, and they have continued into follow-up projects.

All these partnerships have success stories to tell, from greatly improved load balancing on leadership-class machines, to new DFT solver technologies, to dramatically improved algorithms for optimization of functionals, to eigenvalues and eigenfunctions of extremely large matrices, and more.

The SciDAC program aims at transformative science, and this goal has been fulfilled by the new capabilities stemming from UNEDF. But the outcomes reach beyond the many compelling nuclear physics calculations. UNEDF has changed for the better the way that low-energy nuclear theory is carried out, analogous to the shift in experimental programs, moving from many small groups working independently to large-scale collaborative efforts.

\section{Science}

The territory of UNEDF science is the chart of the nuclides in the $(N, Z)$-plane shown in Fig. 2. On this chart, stable nuclei are represented by black squares, while the yellow squares indicate unstable nuclei that have been seen in the laboratory. The sizable green area marked "terra incognita" is populated by unstable isotopes yet to be explored. Above the table of nuclides are shown three broad classes of theoretical methods, which are also used in other fields dealing with strongly interacting many-body systems, such as quantum chemistry and condensed matter physics. Light nuclei and their reactions can be computed by using ab initio techniques (quantum Monte Carlo, nocore shell model) described in Sec. 2.1. Medium-mass nuclei can be treated by configuration interaction (CI) techniques (Sec. 2.2). The bulk of the nuclides are covered by the nuclear DFT described in Sec. 2.3. which provides the theoretical underpinning and computational framework for building a nuclear EDF. Time-dependent phenomena involving complex nuclei, including nuclear reactions, can be described by means of approaches going beyond static DFT (Sec. 2.4). By enhancing and exploiting the overlaps with ab initio and CI approaches, the goal is to construct and validate a nuclear EDF informed by microscopic interactions as well as experimental data.

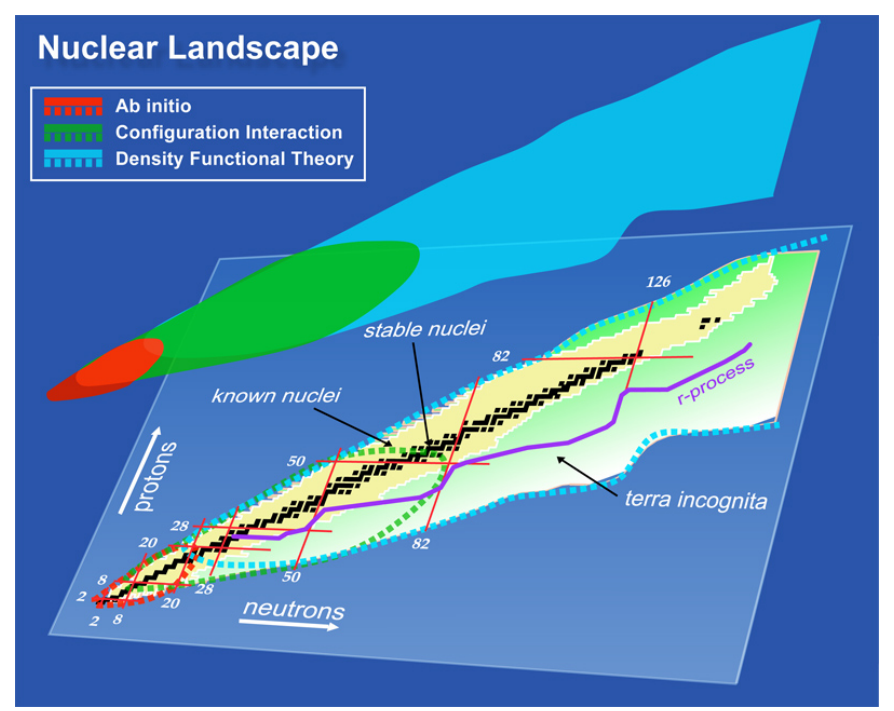

Figure 2: Theoretical approaches for solving the nuclear quantum many-body problem used by UNEDF. The lightest nuclei can be computed by using ab initio methods based on the bare internucleon interactions (red). Medium-mass nuclei can be treated by configuration interaction techniques (green). For heavy nuclei, the density functional theory based on the optimized energy density functional is the tool of choice. (From [1.)

\subsection{Ab initio methods and benchmarking}

$\mathrm{Ab}$ initio methods solve few- and many-body problems by using realistic two- and three-nucleon interactions and obtain the structure and dynamic properties of nuclei. The nuclear interaction depends on the spatial, spin, and isospin coordinates of the nucleons. Consequently, calculations are much more computationally demanding than typical quantum problems. Items of interest include nuclear spectra, charge and magnetic ground-state and transition densities, electron and neutrino scattering, and lowenergy reactions. The main goals are to reproduce known nuclear properties and predict properties that are difficult or impossible to measure.

Several ab initio methods have been developed for studying light nuclei; all have analogues in the study of condensed matter and electronic systems. Quantum Monte Carlo (QMC) methods, including Green's function Monte Carlo (GFMC), use Monte Carlo evaluations of path integrals, explicitly summing over the spin states and isospin states of the system. The most recent GFMC calculations have concentrated on the ${ }^{12} \mathrm{C}$ nucleus, a fascinating system with a low-lying excited $0^{+}$state, the Hoyle state, very near the threshold of three-alpha particles. QMC methods have also been used to calculate the properties of neutron matter and neutrons in inhomogeneous potentials.

No-core shell model (NCSM) methods, including the large-scale many-fermion dynamics nuclear (MFDn) code, expand the interacting states in products of single-particle states and project the low-lying states through large-scale matrix operations. MFDn calculations have been used, for example, to explain the long lifetime of the ${ }^{14} \mathrm{C}$ nucleus 
used in carbon dating. A combination of no-core shell model techniques with the resonating group method is currently used to calculate important low-energy nuclear reactions.

The coupled-cluster method is an ideal microscopic approach to describe nuclei with closed (sub)shells and their neighbors. It exhibits a low computational cost (scales polynomially with system size) while capturing the dominant parts of correlations in the wave function. This method has been employed to describe and predict the structure and reactions of neutron-rich oxygen and calcium isotopes.

\subsection{1. $G F M C$}

Green's function Monte Carlo calculations start with an initial trial state $\Psi_{T}$ and obtain expectation values in the exact eigenfunction $\Psi_{0}$ of the Hamiltonian. These calculations are done by evolution in imaginary time $\tau$ : $\Psi_{0}=\exp [-H \tau] \Psi_{T}$ for sufficiently large $\tau$. The evolution is done in many small steps of $\tau$, each step being a nested 3A-dimensional integral. GFMC was introduced in light nuclei [3, 4] to include the strong correlations induced by the nuclear interaction. This method has been used to calculate the spectra of light nuclei up to ${ }^{12} \mathrm{C}$ [4, 5], as well as form factors, electron scattering, and low-energy reactions 6.

Calculations of ${ }^{12} \mathrm{C}$ require the largest-scale computers available, using a combination of efficient load-balancing for the Monte Carlo and large-scale linear algebra for the spin-isospin degrees of freedom. The calculations of ${ }^{12} \mathrm{C}$ required the development of the Asynchronous Dynamic Load Balancing (ADLB) library to efficiently perform the load balancing on more than 100,000 cores [5].

A program, AGFMC, has been developed over the past 15 years to carry out these calculations [7, 8, 9]. It is a large (80,000 lines) Fortran code that originally used MPI to manage parallelism. At the beginning of this project, the AGFMC code was scaling well up to around 2,000 processes and performing satisfactorily on IBM's Blue Gene/L computer. At that time it was becoming apparent that if the code were to be able to take advantage of new, petascale machines expected to come on line during the five-year project to investigate larger nuclei, a significant increase in the degree of parallelism would need to be incorporated into its main algorithms. The greater degree of parallelism (from thousands to tens of thousands of processes) would give rise to load-balancing problems that would strain the then-used approach.

One of the goals of UNEDF was to construct a software library, intrinsically general-purpose but with features driven by the requirements of AGFMC, to attack the load-balancing problem. The purposes of the library were to supply a programming interface that would enable relatively straightforward migration of the existing AGFMC code to the new load-balancing library and to scale the entire system to much larger degrees of parallelism.

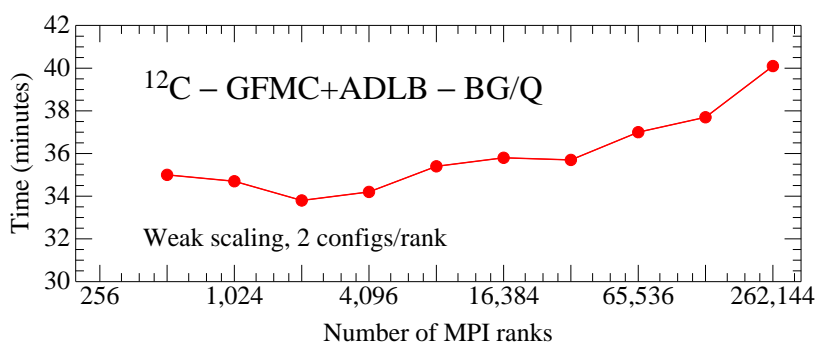

Figure 3: Weak scaling of AGFMC with ADLB in terms of MPI ranks. There are 8 ranks per BG/Q node; each rank is using 6 OpenMP threads. Note the compressed vertical scale.

The result is the ADLB library [5]. ADLB generalizes the classical manager-worker parallel programming model by allowing application processes (workers) to put arbitrary independent work units into a shared pool and get them out to complete them, notifying other processes when they have done so. Work units are assigned types and priorities by the workers and retrieved according to these properties, allowing complex algorithms to be implemented, despite the simple nature of the parallel programming model. Scalability is achieved by dedicating a small percentage (but still potentially a large number) of the job's processes to maintaining this work pool and responding to put and get requests. These "server" processes execute independently from the application processes, thus allowing asynchronous load balancing of process load, memory consumption for the work pool, and message traffic.

This scheme has worked well. Most of the MPI programming in the original AGFMC code has been absorbed into the ADLB library, yet the overall code structure has been maintained. Scalability has been extended to more than 32,000 processes on BG/P and more than 260,000 processes on BG/Q (see Fig. 3), enabling scientific results unattainable before this project was undertaken.

The ${ }^{12} \mathrm{C}$ nucleus is particularly intriguing because it has a low-lying $0^{+}$excited state (the "Hoyle" state) very near the energy of the breakup into three alpha particles. This state is essential for the nucleosynthesis of carbon in stars through the triple-alpha process. For ${ }^{12} \mathrm{C}$ the $\Psi_{T}$ are linear combinations of shell-model and alpha-cluster states. Figure 4 shows the convergence of the calculations of the ground and Hoyle states in the AGFMC calculations. Two different sets of initial states are propagated to $\tau \approx 1.0 \mathrm{MeV}^{-1}$; they yield consistent results. The groundstate energy is well reproduced, and the Hoyle state excitation energy is approximately reproduced (see [10, 11] for complementary calculations of the Hoyle state). The ground-state form factor of ${ }^{12} \mathrm{C}$ is also reproduced by these calculations.

Other recent applications of AGFMC include pair momentum distributions [12, electromagnetic transitions [13, and the studies of trapped neutrons ("drops") described in Sec. 2.3.4 


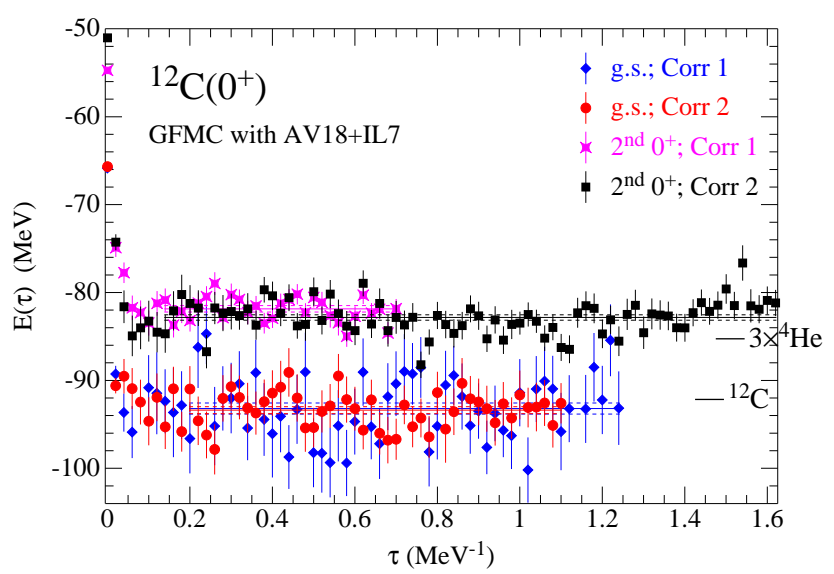

Figure 4: Convergence of the ground state (lower curves) and Hoyle state (upper curves) for different initial states as a function of imaginary time.

\subsubsection{NCSM and $\mathrm{MFD} n$}

The measured lifetime of ${ }^{14} \mathrm{C}, 5730 \pm 30$ years, is a valuable chronometer for many practical applications ranging from archeology to physiology. It is anomalously long compared with lifetimes of other light nuclei undergoing the same decay process, allowed Gamow-Teller (GT) beta decay. This lifetime poses a major challenge to theory because traditional realistic nucleon-nucleon (NN) interactions alone appear insufficient to produce the effect [14]. Since the transition operator, in leading approximation, depends on the nucleon spin and charge but not the spatial coordinates, this decay provides a precision tool to inspect selected features of the initial and final nuclear states. To convincingly explain this strongly inhibited transition, we need a microscopic description that introduces all physically relevant 14 -nucleon configurations in the initial and final states and a realistic Hamiltonian.

Since the nuclear strong interaction governs the configuration mixing, the Hamiltonian matrix eigenvalue problem is a very large, sparse matrix in the configuration space of 14 nucleons. We address this computational challenge with the MFDn code [16, 17, 18, 19. Aided by a collaboration with applied mathematicians on scalable eigensolvers and computational resources on leadership-class machines, we are able to solve this beta decay problem with sufficient accuracy to resolve the puzzle: the decay is inhibited by the role of 3-nucleon forces (3NFs) as shown in Fig. 5 (see 20] for complementary calculations).

We obtained our results on the Jaguar supercomputer (see Sec.4) using up to 35,778 hex-core processors $(214,668$ cores) and up to 6 hours of elapsed time for each set of low-lying eigenvalues and eigenvectors. The number of nonvanishing matrix elements exceeded the total memory available and required matrix element recomputation "on the fly" for the iterative diagonalization process employing the Lanczos algorithm.

These calculations and many other achievements [21] were made possible by dramatic improvements to MFDn

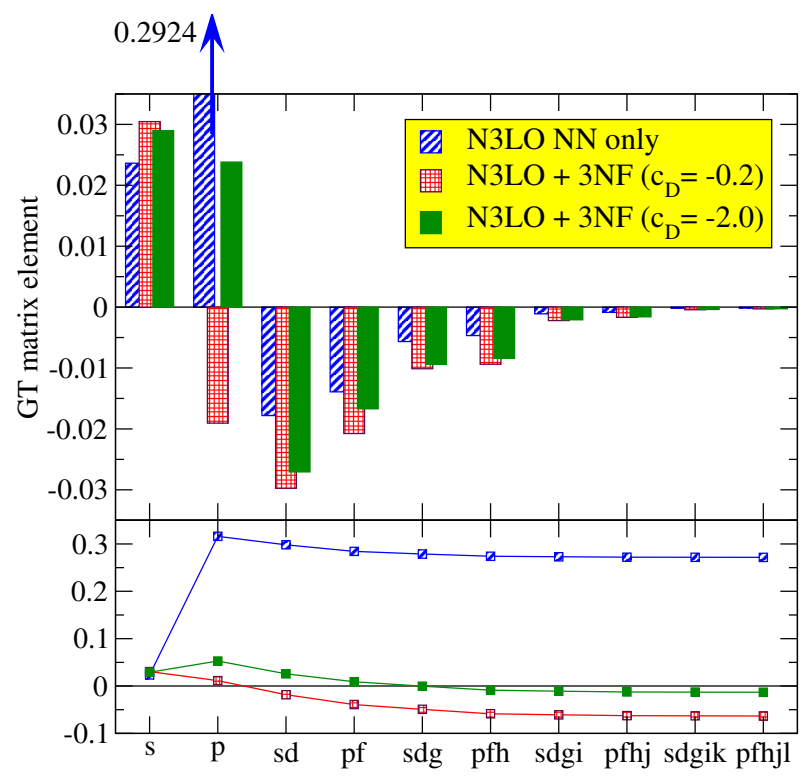

shell

Figure 5: Contributions to the ${ }^{14} \mathrm{C}$ beta decay matrix element as a function of the harmonic oscillator shell when the nuclear structure is described by a chiral effective field theory interaction (adopted from [15]). The top panel displays the contributions with (two right bars of each triplet) and without (leftmost bar of each triplet) the $3 \mathrm{NF}$ at $N_{\max }=8$. Contributions are summed within each shell to yield a total for that shell. The bottom panel displays the running sum of the GT contributions over the shells. Note the order-of-magnitude suppression of the $0 p$-shell contributions arising from the 3NFs.

capabilities during the UNEDF project 22 . The current scaling performance of MFDn is demonstrated in Fig. 6. Other recent applications of MFDn include the prediction (before experimental confirmation) of the spectroscopy of proton-unstable ${ }^{14} \mathrm{~F} 23$ and studies of trapped neutrons ("drops") with a variety of interactions and other ab initio computational methods 24.

\subsubsection{NCSM and the resonating group method}

Weakly bound nuclei, or even unbound exotic nuclei, cannot be understood by using only bound-state techniques. Our ab initio many-body approach, no-core shell model with continuum (NCSMC), focuses on a unified description of both bound and unbound states. With such an approach, we can simultaneously investigate structure of nuclei and their reactions. The method combines square-integrable harmonic-oscillator basis (i.e., via the NCSM [21]) accounting for the short- and mediumrange many-nucleon correlations with a continuous basis (i.e., via the NCSM with the resonating group method (NCSM/RGM) [25, 26]) accounting for long-range correlations between clusters of nucleons. With this technique, we can predict the ground- and excited-state energies of light nuclei ( $p$-shell, $A \leq 16$ ) as well as their electromagnetic moments and transitions, including weak transitions. Furthermore, we can investigate properties of resonances and calculate characteristics of binary nuclear reactions (e.g., 


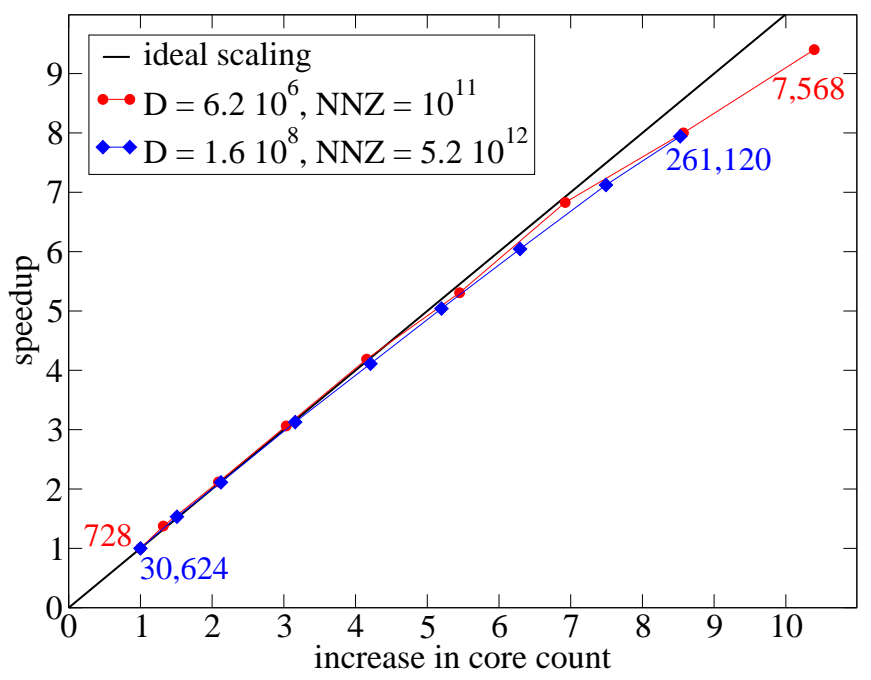

Figure 6: Strong scaling for MFDn: speedup for 500 Lanczos iterations (the most time-consuming phase of the code). Two problems are shown with their dimension (D) and number of nonzero matrix elements (NNZ) in the legend. The smaller is ${ }^{7} \mathrm{Li}(\mathrm{D}=6.2$ million, $\mathrm{NNZ}=118$ billion), and the larger is ${ }^{10} \mathrm{~B}(\mathrm{D}=160$ million, $\mathrm{NNZ}=5.2$ trillion). The smaller problem needs at least $1 \mathrm{~TB}$ in order to store all nonzero matrix elements in core and needs, therefore, at least 728 cores to fit the problem in core. The larger problem needs at least 42 TB, and we used between 30,624 and 261,120 cores for that problem.

cross sections, analyzing powers).

Recent applications of our ab initio techniques include an investigation of the unbound ${ }^{7} \mathrm{He}$ [27, calculations of ${ }^{3} \mathrm{H}(d, n){ }^{4} \mathrm{He}$ and ${ }^{3} \mathrm{He}(d, p){ }^{4} \mathrm{He}$ fusion 28 (see Fig. 7), and calculation of the ${ }^{7} \mathrm{Be}(p, \gamma)^{8} \mathrm{~B}$ radiative capture [29, which is important for the standard solar model and neutrino physics (see Fig. 8). We also developed a three-cluster extension of the method to describe the Borromean nuclei (e.g., ${ }^{6} \mathrm{He}$ and ${ }^{11} \mathrm{Li}$ ).

\subsubsection{Coupled-cluster method}

The coupled-cluster method 30, 31, 32, 33, exhibits a favorable scaling that grows polynomially with the mass number of the nucleus and the size of the model space. The UNEDF collaboration employed an $m$-scheme-based coupled-cluster code [34] and an angular-momentum coupled code 35. The latter exploits the preservation of angular momentum and pushed ab initio computation with "bare" interactions from chiral effective field theory [36] to medium-mass nuclei [37. Coupled-cluster theory is based on a similarity-transformed Hamiltonian and employs a nontrivial vacuum such as the Hartree-Fock state. In practice, one iteratively solves a large set of nonlinear coupled equations. The exploitation of rotational invariance considerably reduces the number of degrees of freedom but comes at the cost of working in a much more complicated scheme (involving angular momentum algebra) that poses challenges for a scalable and load-balanced implementation.

During UNEDF, several conceptual advances in physics and computing were made with the coupled-cluster

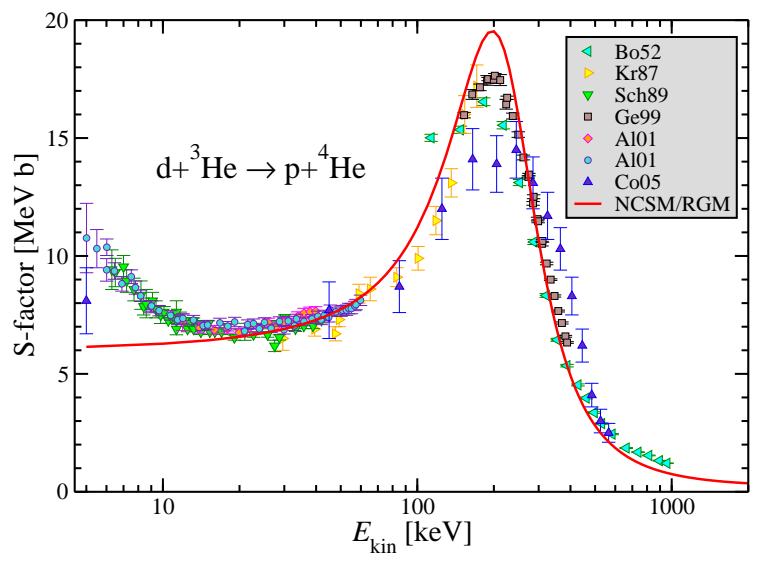

Figure 7: Experimental results for S-factor of ${ }^{3} \mathrm{He}(d, p){ }^{4} \mathrm{He}$ reaction from beam-target measurements. The full line represents the ab initio calculation. No low-energy enhancement is present in the theoretical results, contrary to the laboratory beam-target data represented by symbols; see [28] for details.

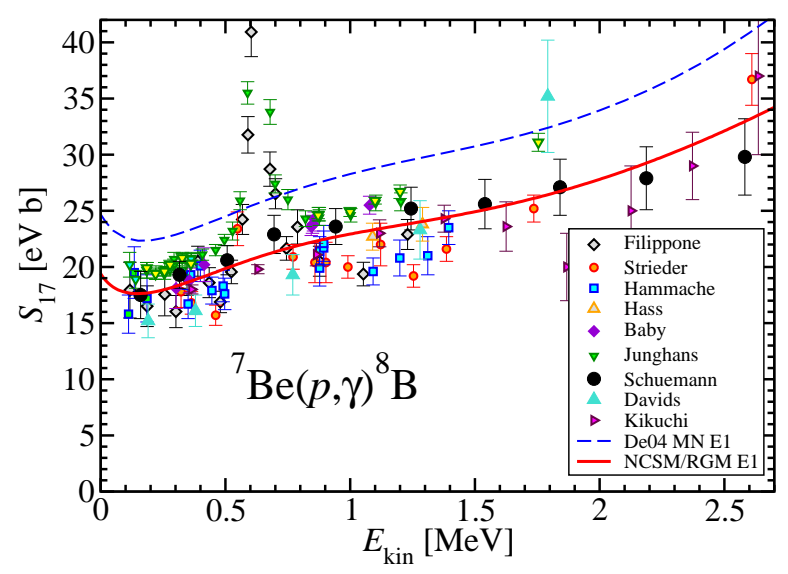

Figure 8: Ab initio calculated ${ }^{7} \mathrm{Be}(p, \gamma)^{8} \mathrm{~B}$ S-factor (solid line) compared with experimental data and the calculation used in the latest evaluation (dashed line); see [29] for details.

method. On the physics side, these include the angularmomentum coupled implementation of the coupled-cluster method [37, the use of a Gamow basis for computation of weakly bound nuclei [38, 39, a practical solution to the center-of-mass problem in nuclear structure computations [40, the extension of the method to nuclei with up to two nucleons outside a closed subshell [41, the approximation of three-nucleon forces as in-medium correction to nucleon-nucleon forces [42, 43, 44, and the development of theoretically founded extrapolations in finite oscillator spaces [45]. On the computational side, scal- 


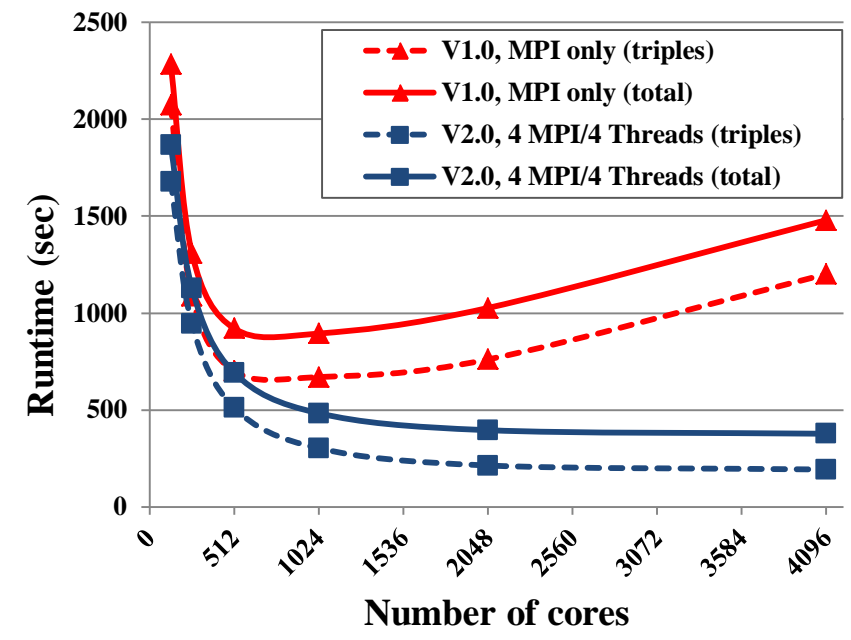

Figure 9: Comparison of runtime for ${ }^{40} \mathrm{Ca}$ in 12 oscillator shells using MPI only V1.0 and hybrid MPI/OpenMP V2.0. Solid lines show total runtime; dashed lines show runtime of triples calculation only.

ing was improved by a work-balancing approach [46, 47] based on MPI and OpenMP such that the model-space size has increased from ten oscillator shells at the inception of UNEDF 48] to 20 oscillator shells at UNEDF's completion 44. Figure 9 shows how adding the use of MPI and OpenMP in V2.0 improved the code's scalability to thousands of cores, beyond a few hundred cores in V1.0 using MPI only, when calculating the small system of ${ }^{40} \mathrm{Ca}$ in 12 oscillator shells. We note that the number of singleparticle orbitals grows as the third power with the number of oscillator shells and that the number of computational cycles - in the coupled-cluster method with singles and doubles (CCSD) approximation - grows as $n_{\mathrm{o}}^{2} n_{\mathrm{u}}^{4}$ (where $n_{\mathrm{o}}$ and $n_{\mathrm{u}}$ are the numbers of occupied and unoccupied single-particle states, respectively). Thus, conceptual and algorithmic improvements during UNEDF allowed us to solve problems that naïvely required an increase of computational cycles by about a factor 4,000. The combined efforts culminated in the computation of neutron-rich isotopes of oxygen 44 and calcium [49].

Doubly magic nuclei are the cornerstones for our understanding of entire regions of the nuclear chart within the shell model. For this reason, studies on the evolution of structure in neutron-rich semi-magic isotopes of oxygen, calcium, nickel, and tin are central to experimental and theoretical efforts. With ${ }^{40,48} \mathrm{Ca}$ being doubly magic nuclei, many studies were aimed at understanding the structure of the rare isotopes ${ }^{52,54} \mathrm{Ca}$ and questions regarding the $N=32,34$ shell closures [50, 51, 52, 53, 54].

A first-principles description of rare calcium isotopes is challenging because it requires the control and understanding of continuum effects (due to the weak binding) and 3NFs (as often pivotal contributions arise at next-to-nextto leading order in chiral effective field theory [55, 56, 57]). Reference [49] reports coupled-cluster results for neutronrich isotopes of calcium that include the effects of the

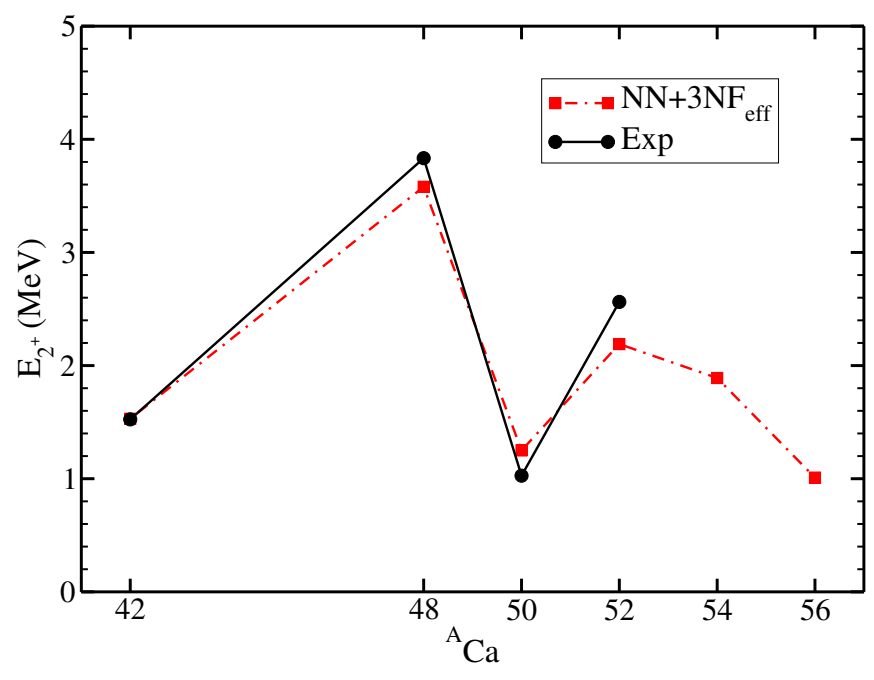

Figure 10: Excitation energies of $J^{\pi}=2^{+}$states in Ca isotopes. The theoretical results (red squares) agree well with data (black circles) and predict a soft subshell closure in ${ }^{54} \mathrm{Ca}$.

continuum and 3NFs (see 58 for complementary calculations). It predicts a soft subshell closure in the $N=32$ nucleus ${ }^{54} \mathrm{Ca}$ and an ordering of single-particle orbitals in neutron-rich calciums that is at variance with naïve shellmodel expectations. Figure 10 shows the computed energies of the first excited $J^{\pi}=2^{+}$state in some isotopes of calcium and compares them with available data. The high excitation energy in ${ }^{48} \mathrm{Ca}$ is due to its double magicity, and the somewhat increased excitation energies in ${ }^{52,54} \mathrm{Ca}$ suggest that these nuclei exhibit a softer subshell closure. Where data are available, the theoretical results agree well with experiment. For ${ }^{54} \mathrm{Ca}$, theory made a prediction that has recently been verified experimentally [59].

\subsection{Configuration interaction}

The nuclear shell model has been very effective in describing the physics of larger nuclei beyond the current reach of pure ab initio methods; indeed, Eugene Wigner, Maria Goeppert-Mayer, and J. Hans D. Jensen were awarded the 1963 Noble prize for the fundamental symmetries and mean field features that underlie the successful nuclear shell model. The shell model for larger nuclei uses the same configuration interaction methods as the NCSM methods described previously, but with more truncated model spaces where not all nucleons are "active" and with effective interactions tailored for these spaces.

Since there are numerous challenging physical applications in nuclear physics that vary across the periodic table, different CI approaches are needed to efficiently exploit the available computational resources. CI approaches developed or improved within UNEDF include the following:

- No-core shell model in the $m$-scheme basis (MFDn [16, 18, 19]; BIGstick 60, 61, 62]). 
- No-core shell model in a coupled angular momentum basis (MFDNJ 63, 64).

- Shell model with a core in a coupled angular momentum basis (NUSHELLX 65, 62]).

UNEDF took advantage of common elements in the various CI approaches to improve the effectiveness of the nuclear shell model for all nuclei.

These CI codes utilize an input NN interaction file and a Coulomb interaction between the protons. They all work in the neutron-proton basis (i.e., break isospin) and allow for charge-dependent NN interactions. In addition, several of these codes accept $3 \mathrm{NFs}$ as input. All these codes evaluate the spectra, wavefunctions, and a suite of observables for low-lying states of the nucleus.

The implemented algorithms differ considerably among the codes as well as support systems for processing the output files generated, such as the wavefunctions and onebody density matrices, both static and transition. Numerous cross-comparisons between the codes have been accomplished and their respective accuracies confirmed. Eigenenergies are obtained to the accuracy of $1 \mathrm{keV}$ or better. Other observables are found to differ at the level of a few percent because of numerical noise in the wavefunctions.

Except for MFDNJ (which followed MFDn), the codes evolved along independent paths, which emphasized various strategic physics and technological goals. For example, the challenges of addressing heavier nuclei impel working with a nuclear core; the challenges of working with leadership-class machines versus local clusters drive some of the algorithmic decisions. The burden of communications and memory restrictions help resolve the challenge of store-in-memory versus recompute-on-the-fly strategies that are implemented differently in these CI codes.

In light of the need to store large amounts of data for retrieval, postanalyses, and reproducibility, we have developed a prototype database management system. This prototype records in the database the metadata of every run. The data referenced in the database may include physical observables, one-body density matrices, and wavefunctions that result from the ab initio codes; such data are typically stored on the platforms where runs are performed. A user can access this database over the web and find out whether the runs of interest have already been performed and where the results may be located.

\subsection{Nuclear density functional theory}

Because of the enormous configuration spaces involved, the properties of complex heavy nuclei are best described by the superfluid nuclear density functional theory 66 - rooted in the self-consistent Hartree-Fock-Bogoliubov (HFB), or Bogoliubov-de Gennes, problem. The main ingredient of nuclear DFT is the effective interaction between nucleons captured by the energy density functional. Since the nuclear many-body problem involves two kinds of fermions, protons and neutrons, the EDF depends on two kinds of densities and currents 67, 68: isoscalar (neutronplus-proton) and isovector (neutron-minus-proton). The coupling constants of the nuclear EDF are usually adjusted to selected experimental data and pseudodata obtained from ab initio calculations. The self-consistent HFB equations allow one to compute the nuclear ground state and a set of quasiparticles that are elementary degrees of freedom of the system and that can be used to construct better approximations of the excited states. The HFB equations constitute a system of coupled integrodifferential equations that can be written in a matrix form as a self-consistent eigenvalue problem, where the dependence of the HFB Hamiltonian matrix on the eigenvectors (quasiparticle wavefunctions) induces nonlinearities.

The atomic nucleus is also an open system having unbound states at energies above the particle emission threshold, and this has implications for the nuclear DFT. The finiteness of the HFB potential experienced by a nucleon implies that the energy spectrum of HFB quasiparticles contains discrete bound states, resonances, and nonresonant continuum states 69]. The size of the continuum space may become intractable, especially for complex geometries where self-consistent symmetries are broken. To this end, one has to develop methods 70 to treat HFB resonances and nonresonant quasiparticle continuum without resorting to the explicit computation of all states.

The application of high-performance computing, modern optimization techniques, and statistical methods has revolutionized nuclear DFT during recent years, in terms of both developing new functionals and carrying out advanced applications. Optimizing the performance of a single $\mathrm{HFB}$ run is crucial for making the EDF optimization [71, 72] manageable and quickly computing tables of nuclear observables [73, 74, 75, 76, in order to assess theoretical uncertainties. These advances are described in the following sections.

\subsubsection{DFT solvers}

Solutions of HFB equations can be obtained either by direct numerical integration on a mesh, provided proper boundary conditions are imposed on the domain, or by expansion on a basis. For the latter case, the harmonic oscillator ( $\mathrm{HO})$ basis proves particularly well-adapted to nuclear structure problems, as it offers analytical, localized solutions with convenient symmetry and separability features. Although solving the HFB equations for a given nuclear configuration is relatively fast on modern computers, accurate characterization of nuclear properties often requires simultaneous computations of many different configurations, from a few dozen (e.g., one-quasiparticle configurations in odd mass nuclei) to a few billion or more in extreme applications (such as probing multidimensional potential energy surfaces of heavy nuclei during the fission process).

The two primary DFT solvers based on HO expansion used by the collaboration are HFBTHO 77] and HFODD 
78; see [79 and 80, respectively, for their latest releases. Both codes solve the HFB equations for generalized Skyrme functionals in a deformed HO basis and have been carefully benchmarked against one another up to the $1 \mathrm{eV}$ level. HFBтно assumes axial and time-reversal symmetry of the solutions, making it a very fast program (execution completes in typically less than 1 minute on a single node). It is particularly suited for EDF optimization (see Sec. 2.3.3) or large-scale surveys of nuclear properties 74, 75]. The solver HFODD is fully symmetry-unrestricted: this versatility is necessary for science applications such as the computation of fission pathways 81 or description of high-spin states 82 .

The new versions of each solver benefited significantly from recent advances in high-performance computing and from collaborations with computer scientists in UNEDF. By expanding the use of tuned BLAS and LAPACK libraries, significant performance gains were reported for both codes and enabled new, large-scale studies 83. The speed of HFBTHO was further improved by a factor of 2 by incorporating multithreading; HFODD was turned into a hybrid MPI/OpenMP program: nuclear configurations are distributed across nodes, while on-node parallelism is implemented via OpenMP acceleration.

Figure 11 illustrates two algorithmic improvements to the DFT solver HFODD. The implementation of the Broyden method for nonlinear iterative problems [85] has reduced substantially the number of iterations needed to converge the solution in practical applications. The second example shows the application of the augmented Lagrangian method (ALM) to fission in ${ }^{252} \mathrm{Fm} \mathrm{[84}$. This method is generally used for constrained optimization problems; it allows precise calculations of multidimensional energy surfaces in the space of collective coordinates. Indeed, while the standard quadratic penalty method often fails to produce a solution at the required values of constrained variables on a rectangular grid, the ALM performs well in all cases. Both improvements displayed in Fig. 11 are key to producing realistic large-scale surveys of fission properties in heavy nuclei on leadershipclass computers, where walltime is limited and expensive.

Another HFB solver developed by UNEDF is HFBAx. It is based on the B-splines representation of coordinate space and preserves axial symmetry and space inversion [86]. The solver has been carefully benchmarked with нFBтно and used in several applications involving complex geometries, such as fission 87 and competition between normal superfluidity and LarkinOvchinnikov (LOFF) phases of polarized Fermi gases in extremely elongated traps [88. Hybrid parallel programming (MPI+OpenMP) has been implemented in HFB-AX to treat large box sizes that are important for weakly bound heavy nuclei.

New generations of DFT solvers will be taking advantage of emerging architectures, such as GPUs, and new programming paradigms. In particular, the cost of performing dense linear algebra in both HFBTHO and HFODD
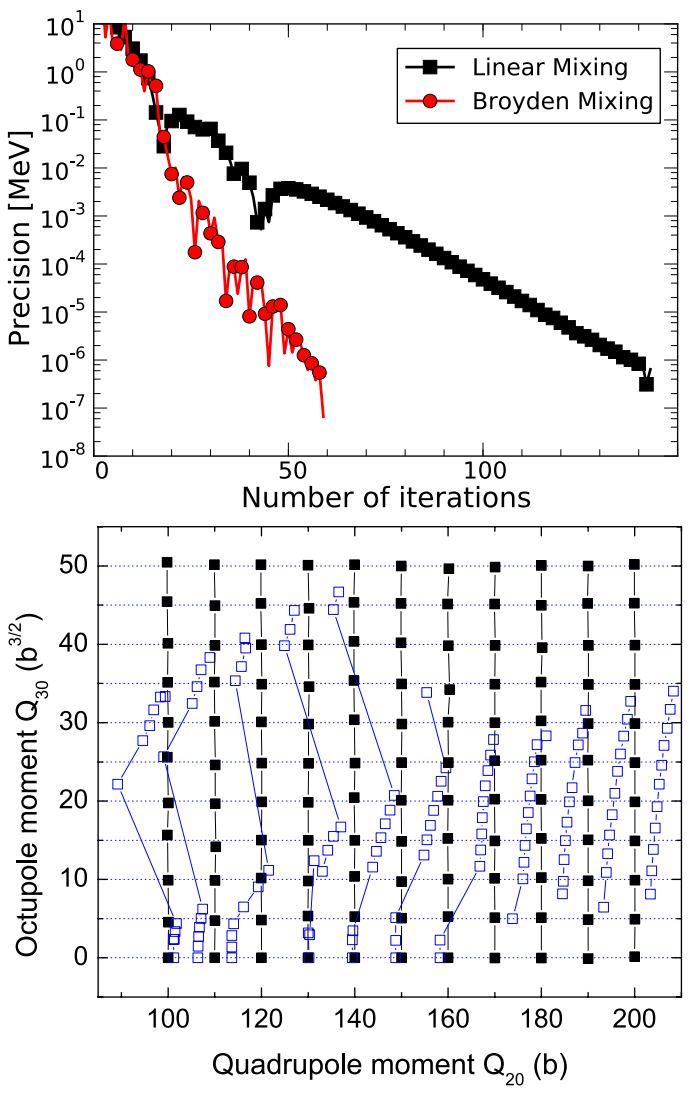

Figure 11: Algorithmic improvements to HFODD. Top: Convergence for a typical HFB calculation in the ground state of ${ }^{166} \mathrm{Dy}$ with HFODD version 2.49t 80. Using the Broyden method to iterate the nonlinear HFB equations has provided significant acceleration compared with traditional linear mixing techniques. Bottom: Comparison between the augmented Lagrangian method (black squares) and the standard quadratic penalty method (open squares) for the constrained HFB calculations of the total energy surface of ${ }^{252} \mathrm{Fm}$ in a two-dimensional plane of elongation, $Q_{20}$, and reflection-asymmetry, Q30. (From 84.)

can become prohibitive as the size of the HO basis increases, especially for more realistic energy functionals involving some form of nonlocality; this necessitates novel techniques to handle many-body matrix elements [89. The massive amount of data generated by large-scale DFT simulations will also require significant investments in visualization and data-mining techniques.

\subsubsection{Multiresolution 3D DFT framework}

A parallel, adaptive, pseudospectral-based solver, MADNESS-HFB, has been developed to tackle the fully symmetry-unrestricted HFB problem for both real and complex wavefunctions in large and asymmetric boxes. The main mathematical and algorithmic advantage of MADNESS-HFB is its multiscale-multiresolution and sparse approximation of functions and the application of operators in coordinate space with guaranteed accuracy but finite precision. MADNESS-HFB prefers to work with functions and operators with pseudo-spectral approximations based on a multiwavelet basis (up to order 30). Since 


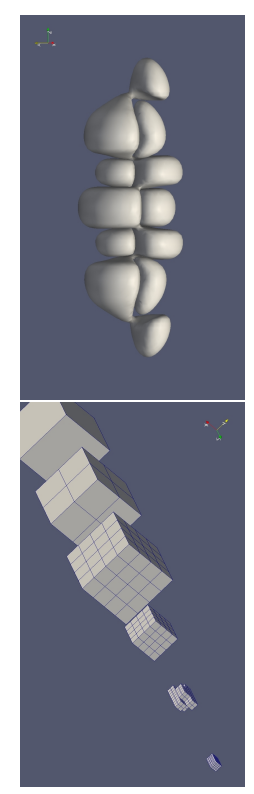

Scaling of MADNESS on CRAY XK-7

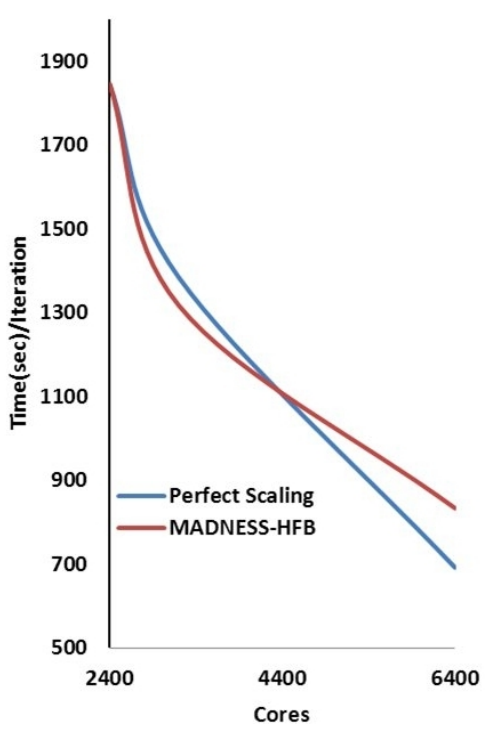

Figure 12: Quasiparticle wavefunction for a DFT simulation (left, top) and its six levels of multiresolution structure (left, bottom). The refinement structure is especially noticeable at levels 5 and 6. Right: The parallel speedup of one iteration of MADNESS-HFB, for solving the DFT problem for 1,640 3D quasiparticle wavefunctions with over 4.4 billion equations and unknowns; this simulation was performed within a box with a spatial dimension of 120 fermis, using 8 multiwavelets, up to level $8+$ of refinement, and with a relative precision of $10^{-6}$.

the multiwavelets consist of smooth, singular, and discontinuous functions with spatial locality (compact support), they are well suited for localized approximation of weak singularities and discontinuities or regions of high curvature 90, 91, 92. Gibbs effects are also reduced. The object-oriented (OO) nature of the software and templatebased programming allow each wavefunction and each integral or differential operator to have its own boundary condition and its own sparse pseudospectral expansion. The usual boundary conditions (e.g., Dirichlet, Neumann, Robin, quasi-periodic, free, and asymptotic conditions) are supported. Fast applications of Green's function for the direct solution of Poisson's equation and the Yukawa scattering kernel are available [93, 94, 95]. In the multiwavelet representation, these approximate Green's functions and their applications are again based on sparse data with guaranteed precision, in contrast to dense tensors based on the use of some other basis sets. Other Green's functions can also be constructed.

If desired, the user can specify solvers and routines from other dense and sparse linear algebra packages such as LAPACK or SCALAPACK. For example, parallel and vectorized adaptive quadrature permit the construction of the Hamiltonian matrix in the usual manner by using the $\ell_{2}$ norm. The Hamiltonian can be diagonalized by using multithreaded LAPACK (or a parallel eigensolver), and the eigenvectors can be converted back to coefficients for the multiwavelet representation. Other capabilities, such as high-order approximation of propagators and timestepping required for the solution of time-dependent DFT, are also available from applications in time-dependent molecular DFT, as well as from simulation of attosecond dynamics [96, 97.

Underlying this mathematical capability is a parallel runtime system that permits the software to scale to hundreds of thousands of processors and runs on platforms from laptops to leadership-class computers. The ability to use laptops and workstations is particularly attractive for model and code development and testing. In addition, the embedding of a parser permits the OO-based $\mathrm{C}++$ templated codes representing operations on the coefficients of each wavefunction to be executed as parallel tasks. This parser permits out-of-order, distributed multithread executions with task- and data-dependency analysis. This reduces the stalling of execution units due to data dependencies. A user-configured and executed parallel load-balancing method is also available, as is a parallel checkpoint and restart method.

The 3D MADNESS-HFB has been benchmarked with the spline-based 2D solver HFB-Ax [86], 3D HFODD [80, and the 1D code HFBRAD 98 for a variety of problems. Because MADNESS-HFB has no limit on the size of the computational domain, we were able to capture quasiparticle wavefunctions with long tails or nonsymmetric potentials with steep curvatures and cut-offs to overcome some of the limitations of the other solvers. The adaptive structure is illustrated in Fig. 12 .

The current MADNESS-HFB approach to the HFB problem is as follows 99. Let the coefficients of the wavefunctions in the tensor product multiwavelet representation be the unknowns. The user provides an initial relative precision, a set of initial wavefunctions (e.g., in terms of the HO basis, splines, etc.), and boundary conditions to start the iterative procedure. All the functions, potentials, operators, and expansion lengths are adaptively represented as needed by the user-defined precision. A generalized matrix eigenvalue problem is formed by adaptive quadrature. The solution eigenvectors are converted to a sparse multiwavelet representation for updating the lengths of the expansion and the coefficients in the potentials, gradients, and other terms before the next iteration and diagonalization. The speed and performance depend on the number of coefficients. Usually, the simulations begin with a low relative precision, to capture the low-order terms quickly, before adaptively increasing the order of approximation and the precision for more accurate results.

\subsubsection{EDF optimization}

One of the focus areas of UNEDF was the development of an optimization protocol for determining the coupling constants of nuclear EDFs. In particular, the collaboration paid special attention to estimating the errors associated with such a procedure and exploring the correlations among the coupling constants. The UNEDF optimization 
protocol was established by focusing on the Skyrme energy density. We recall that, in this framework, the energy of an even-even nucleus in its ground state is a functional of the one-body density matrix and the pairing tensor. The Skyrme energy density reads

$$
\begin{aligned}
\chi_{t}(\boldsymbol{r})= & C_{t}^{\rho \rho} \rho_{t}^{2}+C_{t}^{\rho \tau} \rho_{t} \tau_{t}+C_{t}^{J^{2}} \boldsymbol{J}_{t}^{2} \\
& +C_{t}^{\rho \Delta \rho} \rho_{t} \Delta \rho_{t}+C_{t}^{\rho \nabla J} \rho_{t} \nabla \cdot \boldsymbol{J}_{t},
\end{aligned}
$$

where the isospin index $t$ labels isoscalar $(t=0)$ and isovector $(t=1)$ densities, $\rho_{t}$ is the one-body density matrix, and $\tau_{t}$ and $\boldsymbol{J}_{t}$ are derived from $\rho_{t}$ [67]. In the pairing channel, we took a density-dependent pairing energy density with mixed surface and volume nature, characterized by the two pairing strengths $V_{0}^{(n)}$ and $V_{0}^{(p)}$ for neutrons and protons, respectively. The set of coupling constants $C_{t}^{u u^{\prime}}$, $V_{0}^{(n)}$, and $V_{0}^{(p)}$ are the parameters $x$ to be determined.

The development of fast DFT solvers (see Sec. 2.3.1), together with the availability of leadership-class computers, permitted us for the first time to set up an optimization protocol at a fully deformed HFB level. Our first parametrization, UNEDF0, was obtained by considering only three types of experimental data: nuclear binding energies of both spherical and deformed nuclei, nuclear charge radii, and odd-even mass differences in selected nuclei [71. After recognizing that deformation properties needed to be better constrained [100, a fourth data type, corresponding to excitation energies of fission isomers in the actinides, was added. The resulting parametrization, UNEDF1, gave a significantly better description of fission properties 72, see Fig. 13 (bottom). With the oncoming UNEDF2 parametrization, we will expand the optimization data set with single-particle level splittings. The new data are expected to better constrain the tensor coupling constants and improve single-particle properties.

Formally, we solve the optimization problem

$$
\min _{x}\left\{\chi^{2}(x)=\sum_{i=1}^{n_{d}}\left(\frac{s_{i}(x)-d_{i}}{w_{i}}\right)^{2}: x \in \Omega \subseteq \mathbb{R}^{n_{x}}\right\},
$$

where $d \in \mathbb{R}^{n_{d}}$ represents the experimental data, $w>0$ represent weights, and the parameters $x$ to be determined are possibly restricted to lie in a domain $\Omega$. This problem is made difficult because some of the derivatives with respect to the parameters $x, \nabla_{x} s_{i}(x)$, may be unavailable for some of the theory simulation observables $s_{i}$.

Traditional approaches for solving (2) in the absence of derivatives typically either estimate these derivatives by finite differencing or treat $\chi^{2}$ as a black-box function of $x$. The former approach can be sensitive to the choice of the difference parameter, and care must be taken that the expense of the differencing does not grow unnecessarily as the number of parameters $n_{x}$ grows. The latter neglects the structure (in the form of the $n_{d}$ residuals) inherent to (2).

In UNEDF, we instead employed a new optimization solver, POUNDers, that exploits the structure in nonlinear

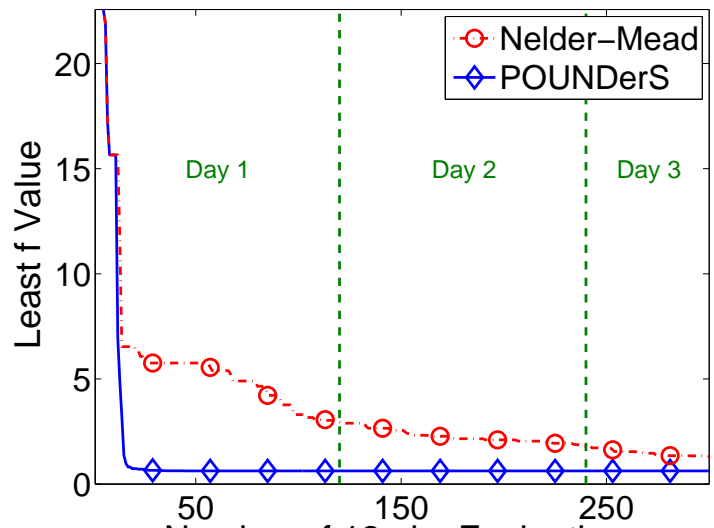

Number of $12 \mathrm{~min}$. Evaluations

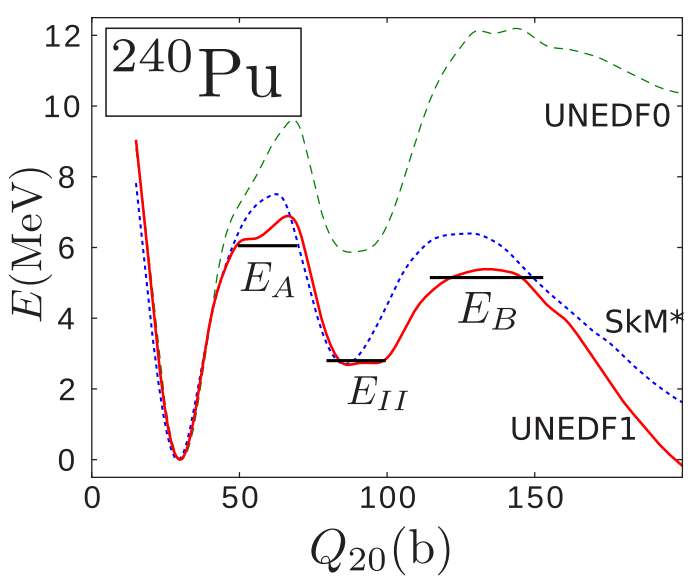

Figure 13: Top: Performance of the Pounders algorithm on the minimization of the $\chi^{2}$ of Eq. 2 as compared with the standard NelderMead method. Bottom: Fission pathway for ${ }^{240} \mathrm{Pu}$ along the mass quadrupole moment $Q_{20}$ calculated with SkM, UNEDF0, and UNEDF1 EDFs. The experimental energy of fission isomer $\left(E_{I I}\right)$ and the inner $\left(E_{A}\right)$ and outer $\left(E_{B}\right)$ barrier heights are indicated [72].

least-squares problems and avoids directly forming computationally expensive derivative approximations. POUNDers follows a model-based Newton-like approach, where the first- and second-order information is inferred by iteratively forming local interpolation models for each residual. Figure 13 (top) shows the efficiency of the solver: not only does it converge faster than the standard NelderMead algorithm, but it also gives a more accurate solution. POUNDers is available in the open-source Toolkit for Advanced Optimization (TAO [101]).

\subsubsection{Neutron droplets and DFT}

The properties of homogeneous and inhomogeneous neutron matter play a key role in many astrophysical scenarios and in the determination of the symmetry energy 102, 103, 104. The equation of state of homogeneous neutron matter has been studied in many earlier investigations (see, e.g., [105]). Since neutron matter is not self-bound, inhomogeneous neutron matter has been theoretically investigated by confining neutrons in external potentials. Although neutron drops cannot be realized in experimental facilities, they provide a model to study neutron-rich 


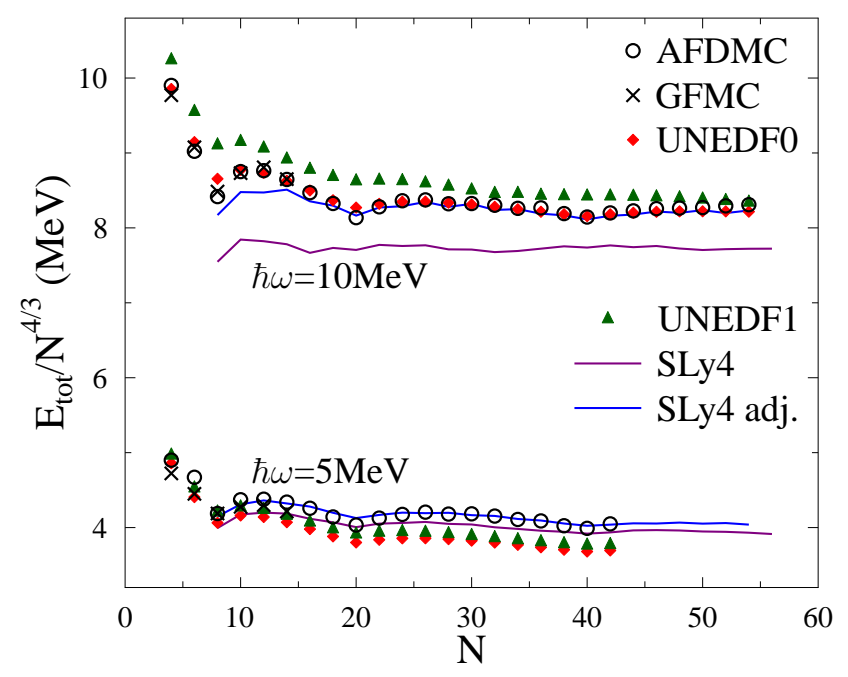

Figure 14: Calculated total energies for neutron droplets in $\hbar \omega=$ $5 \mathrm{MeV}$ and $10 \mathrm{MeV}$ harmonic potentials as a function of the neutron number $N$. The figure shows AFDMC, GFMC, SLy4, and adjusted SLy4 results of [109] together with the UNEDF0 and UNEDF1 results.

isotopes 106, 107, 108, and can bridge ab initio methods and DFT. The external potential confining neutrons has been chosen to change the geometry and density of the system. A Woods-Saxon form produces saturation, making neutron drops similar to ordinary nuclei. Instead, a harmonic potential permits one to better control the calculation of larger systems and to test the approach to the thermodynamic limit.

Nuclear EDFs are commonly optimized to reproduce properties of nuclei close to stability, with close numbers of protons and neutrons. The use of such functionals to study neutron-rich nuclei or the neutron star crust requires large extrapolations in neutron excess. In [109, neutron droplets were studied by using QMC methods starting from a realistic nuclear Hamiltonian that includes the Argonne AV8' two-body interaction supported by the Urbana IX three-body force. This Hamiltonian fits nucleonnucleon phase shifts, gives a satisfactory description of light nuclei, and produces an equation of state of neutron matter that is compatible with recent neutron star observations [110]. The neutron drop's energy calculated by using QMC methods was compared with DFT calculations. The QMC results showed that commonly used Skyrme EDFs typically overbind neutron drops and that this effect is due mainly to the neutron density gradient term. The adjustment of the gradient together with the pairing and spin-orbit terms improves the agreement between ab initio QMC calculations with Skyrme both for the energy and for neutron densities and radii [109].

These results can be compared with the predictions of UNEDF0 and UNEDF1 EDFs. Figure 14 shows the calculated total energies for neutron droplets in $\hbar \omega=5 \mathrm{MeV}$ and $10 \mathrm{MeV}$ harmonic potentials. The auxiliary field diffusion Monte Carlo (AFDMC) and GFMC QMC results
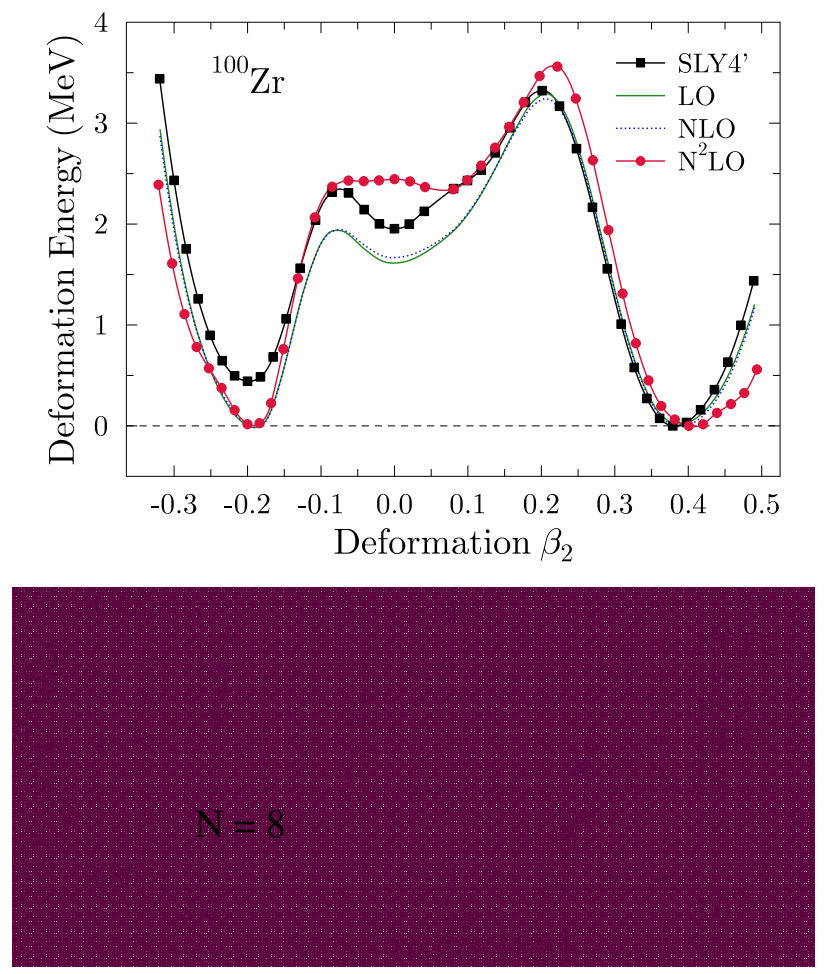

Figure 15: Top: Deformation energy curves for ${ }^{100} \mathrm{Zr}$ calculated using microscopic EDFs derived from chiral EFT interactions at different orders 111. Bottom: Comparison of microscopic EDF calculations of neutron drops at increasing levels of approximation with full NCFC calculations starting from the same Hamiltonian [112.

of [109, calculated with the AV8' + UIX interactions, agree well with the DFT calculations [72]. These are encouraging, since neither UNEDF0 nor UNEDF1 was optimized to the pure neutron matter data. Future EDF optimization schemes will use ab initio results on neutron droplets as pseudodata to improve EDF properties in very neutronrich nuclei.

\subsubsection{Ab initio functionals}

In parallel with efforts to improve the optimization of nuclear EDFs with conventional Skyrme-type terms, UNEDF members sought to construct ab initio functionals based on microscopic chiral effective field theory (EFT) [113. A pathway to such functionals was opened with the development of new renormalization group methods, which led to softer nuclear Hamiltonians, including three-body forces 114. These soft interactions dramatically improve convergence properties in many-body calculations 115, extending the reach of ab initio methods to heavier systems [116, 117, 118. At the same time, they make feasible the construction of a microscopically based EDF using many-body perturbation theory [119] together with improved density matrix expansion (DME) techniques [120, 121, 122. Carrying out this long-term program by individual researchers would be a formidable task, but progress was made possible within UNEDF by 
teaming up with two of the physics-CS/AM partnerships described earlier.

An intermediate step toward a fully ab initio EDF was a new hybrid functional that incorporated long-range chiral EFT interactions to describe pion-range physics and a set of Skyrme-like contact interactions with coupling constants to be fit. The resulting functional has a much richer set of density dependencies than do conventional Skyrme functionals. These were incorporated in the DFT solvers, and new preoptimization procedures were developed by the DFT functional group [111. A proof-of-principle test in the top panel of Fig. 15 shows deformation energies in ${ }^{100} \mathrm{Zr}$ calculated using the DME functional at different orders in the chiral expansion ( $\mathrm{LO}, \mathrm{NLO}, \mathrm{N}^{2} \mathrm{LO}$ ). The deviations from the Skyrme result show nontrivial effects from the finite-range nature of the underlying $\mathrm{NN}$ and $3 \mathrm{~N}$ interactions 111. On-going work includes a rigorous optimization with the procedure outlined in Sec. 2.3.3 and then detailed evaluations of the predictive power of the DME functional.

In order to directly validate the new DME procedures used in 111, it was necessary to benchmark against exact results. The first-ever such calculations were made possible by teaming up with the NCSM-MFDn effort (see Sec. 2.1.2 using neutron droplets as a controlled theoretical test environment as in Sec. 2.3.4. The DME functional was constructed and evaluated for the same (model) Hamiltonian used to generate exact results from MFDn [112 for different numbers of neutrons and varied traps. Figure 15 (bottom) shows the agreement between no-core full configuration (NCFC) results and microscopic EDF calculations at different levels of approximation [112], which validates the optimal strategy used to construct a microscopically based EDF (the points labeled "fit"), while establishing theoretical error bars. Further important DME developments made by external collaborators in the FIDIPRO project [123, 124] will be tested in future investigations.

\subsection{Beyond DFT}

Static DFT provides excellent tools for investigating nuclear binding energies and other ground-state properties. In certain cases, it also can be used to treat dynamical processes. The path to scission during fission, for example, sometimes can be predicted accurately by static DFT. A reliable description of excitation/decay and reactions, however, usually requires methods that go beyond static DFT. Since an ab initio treatment of the nuclear time-evolution is difficult, we employ extensions of DFT and related ideas. The simplest extension, the quasiparticle random phase approximation (QRPA), can be viewed as an adiabatic approximation to the linear response in time-dependent DFT. It provides the entire spectrum of excitations with the same EDF used in static DFT. The adiabatic approximation is, of course, severe (as are the approximations in the density functional itself) but can be applied in any nucleus and folded with reaction theory.
DFT-based QRPA and its applications to nuclear excitation and reactions are discussed in Sec. 2.4.1.

DFT-based methods that go beyond the adiabatic approximation are also now in use. One can exploit the relatively simple dynamics of Fermi gas systems to construct an approximate time-dependent extension of DFT, the time-dependent superfluid local density approximation (TDSLDA). The approximation and related computational techniques can be applied to such classic problems as photoabsorption but also to other time-dependent processes that go beyond linear response. The TDSLDA and its applications are discussed in Sec. 2.4.2.

We also need efficient methods to accurately compute average properties of excited states, such as spin- and parity-dependent level densities, which suffice to treat reactions that proceed primarily through a compound nucleus. Obtaining these densities through a direct diagonalization of the nuclear Hamiltonian and a subsequent level counting is not efficient, but several techniques based on statistical spectroscopy can be used instead. However, even statistical spectroscopy poses computational challenges that demand high-performance computational techniques and resources. Some advances in computational spectroscopy, leading to the first accurate calculation of densities of levels with unnatural parity, are described in Sec. 2.4 .3

\subsubsection{QRPA and reactions}

Members of the UNEDF collaboration developed and exploited both an extremely accurate spherical Skyrme QRPA code [125] and an equally accurate, though computationally much more intensive, deformed (axially symmetric) Skyrme QRPA code [126]. The latter, which can treat both spherical and deformed nuclei, is at the forefront of the modern QRPA. Other groups have developed their own versions of the deformed Skyrme, Gogny, or relativistic QRPA [127, 128, 129, 130, 131]; most of these have some disadvantages compared with ours (e.g., a lack of full self-consistency, oscillator bases that don't capture continuum physics, etc.) but also the occasional advantage (e.g., full continuum wavefunctions rather than the approximate representation of the continuum we describe below).

Both our spherical and deformed codes diagonalize the traditional QRPA A-B matrix [132], constructed from single-quasiparticle states in the canonical basis [132] in a large box (typically $20 \mathrm{fm}$ in each coordinate), so that continuum states are taken into account in discretized form. Both codes work with arbitrary Skyrme density functionals plus delta pairing, include all rearrangement terms, and break neither parity nor time-reversal symmetries. Both output transition amplitudes to the entire spectrum of excited states.

The two codes have some differences as well. The spherical code gets its single-quasiparticle wavefunctions, represented on an equidistant mesh, from an HFB program called HFBMARIO, which derives from the code HFBRAD 
69. The deformed code takes its wavefunctions from the Vanderbilt HFB program 133, which uses B-splines to represent wave functions. Each QRPA code represents those wavefunctions in the same manner as the HFB programs it relies on.

Both QRPA codes have been tested in many ways, including against one another. With the spherical code, we calculated energy-weighted sums in $\mathrm{Ca}, \mathrm{Ni}$, and $\mathrm{Sn}$ isotopes from the proton drip line through the neutron drip line for $J^{\pi}=0^{+}, 1^{-}$, and $2^{+}$multipoles with Skyrme parameter sets $\mathrm{SkM}^{*}$ and SLy4 and found excellent agreement with analytical values 125 . Spurious states in the $J^{\pi}=0^{+}, 1^{+}$, and $1^{-}$channels are well separated from physical states in both codes, though the spherical one performs a bit better because it can include all combinations of HFB two-quasiparticle states in the QRPA basis without making the calculation intractable.

The collaboration used the spherical QRPA to study systematics of $2^{+}$states across the table of isotopes and for microscopic calculations of reaction rates; they used the deformed version for a more limited study of $2^{+}$states and giant resonances in rare-earth nuclei [134.

The collaboration also used transition densities from the spherical QRPA to calculate nucleon-nucleus scattering. The transition amplitudes produced by our spherical matrix QRPA, when combined with single-particle wave functions, yield radial transition densities. These can in turn be folded with the interaction between the projectile and the nuclear constituents (i.e., the nucleon-nucleon interaction) to produce transition potentials that excite target states. References [135, 136, 137] report the development of a code to fold the densities for all QRPA states below $30 \mathrm{MeV}$ with a Gaussian-shaped nucleon-nucleon potential. The result is a microscopic coupled-channels calculation that successfully produces angular distributions and inelastic cross sections for nucleon-induced reactionsquantities that can be compared directly with scattering data - at scattering energies between 10 and $70 \mathrm{MeV}$. To satisfactorily describe observed absorption, we had to explicitly couple also to all one-nucleon pickup channels leading to intermediate deuteron formation. Figure 16 illustrates the effect of such couplings on nucleon-induced absorption cross sections. The direct connection between the calculated cross sections and the nuclear structure ingredients makes this kind of reaction calculation a good test of the structure model.

The collaboration also took significant steps to develop a much more efficient implementation of the QRPA. The finite amplitude method [140, 141] allows one to effectively take the derivatives of mean fields that enter the QRPA equations numerically, through relatively straightforward modifications to the mean-field codes themselves. A simple iterative procedure then solves the equations. Our initial application, to monopole resonances in the deformed nucleus ${ }^{240} \mathrm{Pu} 142$, consumes a small fraction of the time our matrix QRPA implementation would use (see [143, 144] for complementary work based on iterative Arnoldi diagonal-

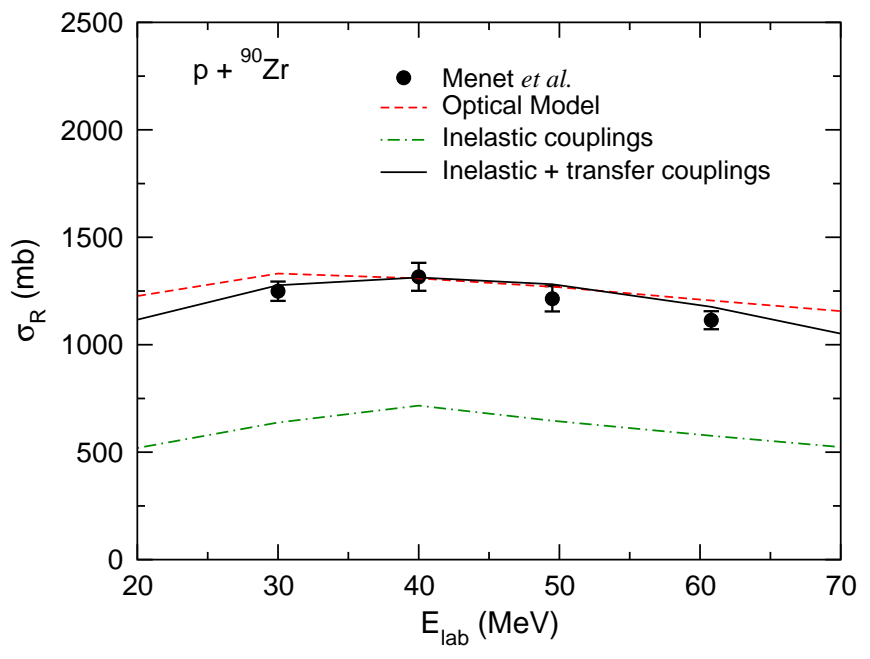

Figure 16: Reaction cross section as a function of incident energy for $p+{ }^{90} \mathrm{Zr}$. The results are shown for couplings to the inelastic states (dash-dotted line) and to the inelastic and transfer channels with nonorthogonality corrections (solid line). The KoningDelaroche [138] optical model calculations are also shown (dashed line). (Data from 139.)

ization).

\subsubsection{Time-dependent DFT for superfluid systems}

The application of DFT to nuclear physics requires two nontrivial elements: the ability to describe both superfluidity and time-dependent phenomena. In order to avoid the nonlocal character of the DFT extension to superfluid systems, the superfluid local density approximation (SLDA) and its time-dependent extension TDSLDA have been developed [145, 146, 147, 148, 149, 150, 151, 152, 153, 154 .

SLDA and TDSLDA have been applied to a large number of fermionic systems and phenomena: vortex structure in neutron matter and cold atomic systems, generation and dynamics of quantized vortices and their crossing and reconnection, excitation of the Anderson-Higgs modes, the LOFF phase, quantum shock waves and excitation of domain walls, one- and two-nucleon separation energies, giant dipole resonance in superfluid triaxial nuclei, and complex collisions. In Fig. 17, we illustrate the case of a headon collision of two superfluid fermion clouds, which was studied experimentally. Both SLDA and TDSLDA are derived by using appropriately determined EDFs with QMC input for homogeneous systems and validating the predictions on independent QMC calculations of inhomogeneous systems in the well-studied case of a unitary Fermi gas; see [147, 148, 150] for details. The form of the EDF for a unitary Fermi gas is largely determined by dimensional arguments; translational, rotational symmetry, and parity; gauge and Galilean covariance (which specifies the dependence on current densities); and renormalizability of the TDSLDA formalism.

For nuclear systems we lack ab initio results of the same 

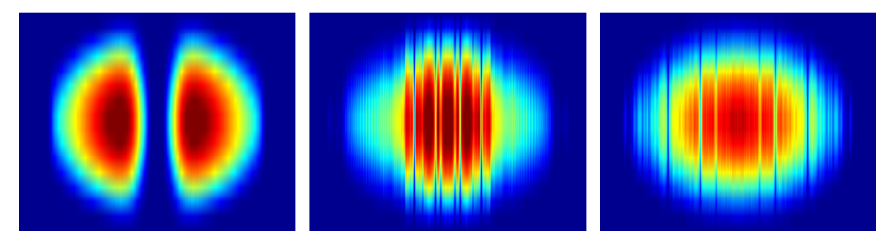

Figure 17: Three consecutive frames of the head-on collision of two fermion clouds of $\approx 750$ particles in which quantum shock waves and domain walls/solitons (topological excitations) are formed 152 . The $x$ - and $y$-directions have an aspect ratio of $\approx 30$.

quality and rely on a more phenomenological approach, but with significant microscopic input. The nuclear EDFs should satisfy the usual symmetries 68, and the consistency with the best available ab initio results.

The numerical implementation of the SLDA and TDSLDA equations leads to hundreds of thousands of coupled nonlinear 3D time-dependent PDEs, which are solved by using the discrete variable representation approach [155, 156] on desktops [147, 148, 149, 150] and-as a result of UNEDF collaborations with computer scientistsleadership-class supercomputers [150, 151, 152, 153, 154]. In Fig. 18 we illustrate the first calculation of the photoexcitation of a triaxial superfluid nucleus performed within TDSLDA $\left({ }^{188} \mathrm{Os}\right)$ and two other axially deformed nuclei, as well as a comparison with the absolute experimental data (without any fitting parameters). The determination of the ground-state properties of these nuclei and their subsequent time-evolution required full diagonalizations of Hermitian matrices of sizes up to $5 \cdot 10^{5} \times 5 \cdot 10^{5}$ and solutions of $5 \cdot 10^{5}$ coupled time-dependent 3D PDEs. Further studies of excitation of medium- and heavy-mass nuclei with $\gamma$-rays, neutrons, relativistic heavy ions, and induced nuclear fission are the next steps.

\subsubsection{Level densities}

The properties of the excited states of nuclei are key to reliably describing reactions and decays. One important type of reaction mechanism is the compound nuclear reaction, which can be described with the statistical model of Hauser and Feshbach [157]. The important ingredient entering the Hauser-Feshbach theory is the spin- and parity-dependent nuclear level density (NLD). Experimental information about NLD is limited for stable nuclei and not available for radioactive nuclides of interest for nuclear astrophysics. Therefore, a large effort is underway to accurately calculate NLD, and an interacting shell model approach would be the best model taking into account the relevant many body correlations beyond DFT. A direct approach by direct CI diagonalization and level counting is not feasible because of the exponential increase in CI dimensions. We recently proposed [158] an approach to calculate shell model spin- and parity-dependent NLD using methods of statistical spectroscopy. In addition, we showed 159 how one can improve this approach to calculate the unnatural parity NLD by removing the con-

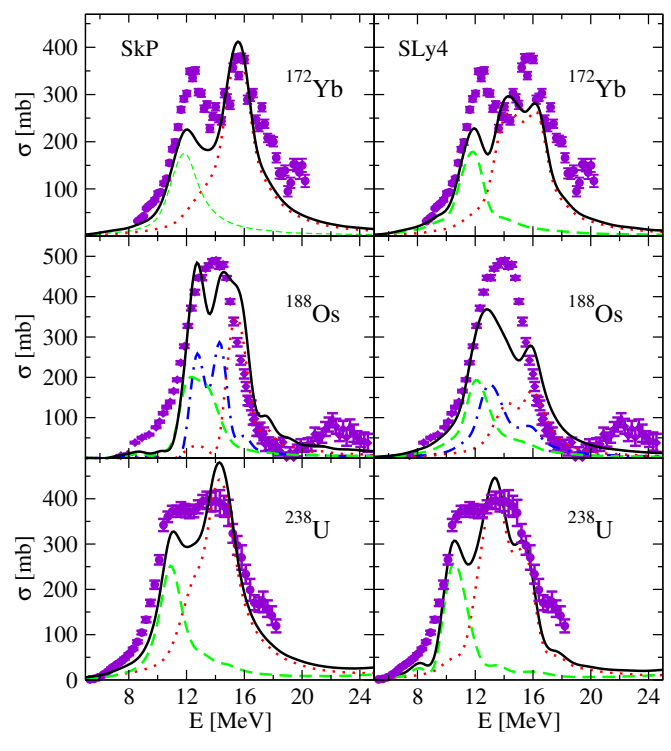

Figure 18: Photoabsorption cross section (solid black line) calculated within TDSLDA using two Skyrme force parameterizations for three deformed open-shell nuclei and the experimental $(\gamma, n)$ cross sections (solid purple circles with error bars); see 153 for details. With dashed (green), dotted (red), and dot-dashed (blue) lines, we display the contribution to the cross section arising from exciting the corresponding nucleus along various symmetry axes.

tribution due to the spurious center-of-mass excitations. The associated algorithms were implemented in a highperformance computer code, JMOMENTS, [160, 161, 162, which runs on massively parallel computers and scales well up to 10,000 processors [160, 162].

Figure 19 shows positive- and negative-parity NLD for ${ }^{26} \mathrm{Al}$ calculated with JMOMENTS compared with the available experimental data obtained by level counting. Some known levels have no clear assignment of the parity, which leads to upper and lower limits. The calculated positiveparity NLD is not new, an $s d$-shell calculation being available for some time. However, the negative-parity NLD was calculated only recently by our approach [161.

\section{Uncertainty Quantification}

Uncertainty quantification is a key element for assessing the predictive power of a model. When working with effective theories with degrees of freedom relevant to the problem, the parameters of the theoretical model often need to be adjusted to the empirical input. To quantify the model uncertainties, sensitivity analysis yields the standard deviations and correlations of the model parameters, usually encoded as a covariance matrix [165, 166, 167, 168.

The calculation of the covariance matrix requires computing derivatives of the observables with respect to the model parameters. When a closed-form expression for the derivatives is not available, we estimate the derivatives numerically using finite differences. To account for the numerical uncertainty associated with the underlying DFT- 


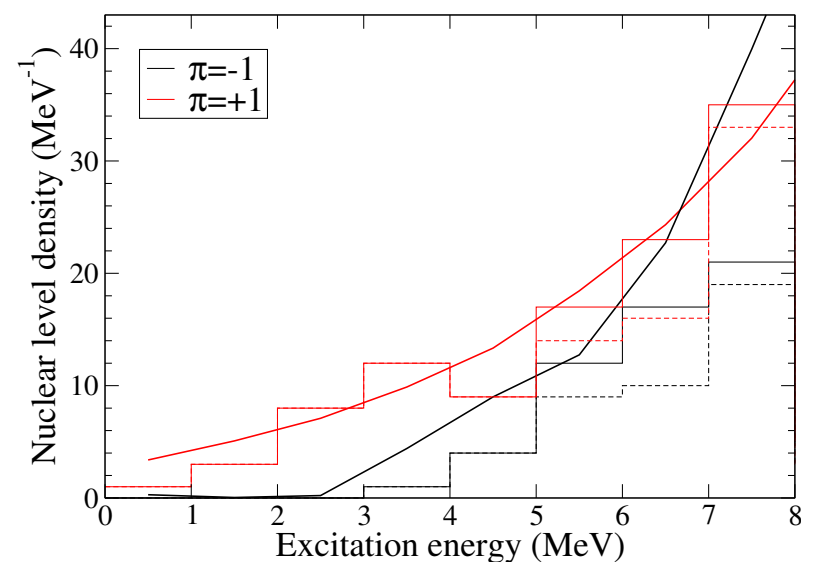

Figure 19: Nuclear-level densities for positive parity (red curve) and negative parity (black curve) of ${ }^{26} \mathrm{Al}$ compared with experimental data; the solid and dotted staircases represent upper and lower limits, respectively. Positive parity NLD is larger than negative parity NLD.

based calculations, we compute the "noise level" of each observable following the approach in 169. The difference parameters used for estimating the Jacobian matrix associated with (2) are then obtained using these noise levels [170].

Uncertainty quantification was one of the key topics of the EDF optimization work performed in the UNEDF collaboration [71, 72]. The upper panel of Fig. 20 shows the UNEDF1 correlation matrix, obtained from the sensitivity analysis. As can be seen, some of the surface parameters of the UNEDF1 EDF are strongly correlated. In 76 we used this information to assess the robustness of the current EDFs in the predictions of the nuclear landscape limits. This is illustrated in the lower panel of Fig. 20 . which shows calculated and experimental two-neutron separation energies for the isotopic chain of even-even zirconium isotopes. The differences between model predictions are small in the region where data exist and grow steadily when extrapolating toward the two-neutron drip line $\left(S_{2 n}=0\right)$. Nevertheless, the consistency between the models was found to be surprisingly good. This study required massive parallel calculations of the nuclear mass tables 75 .

\section{High-Performance Computing Resources}

UNEDF science has benefited from access to some of the largest computers in the world, provided primarily by DOE's Innovative and Novel Computational Impact on Theory and Experiment (INCITE) program [171. In particular, the largest computations of UNEDF were carried out on the "Jaguar" machine at Oak Ridge National Laboratory and the "Intrepid" machine at Argonne National Laboratory. Jaguar has gone through several processor upgrades during the project, taking it from 30,976 cores (Cray XT4 in 2008) to 298,592 cores (Cray XK6 in 2012);
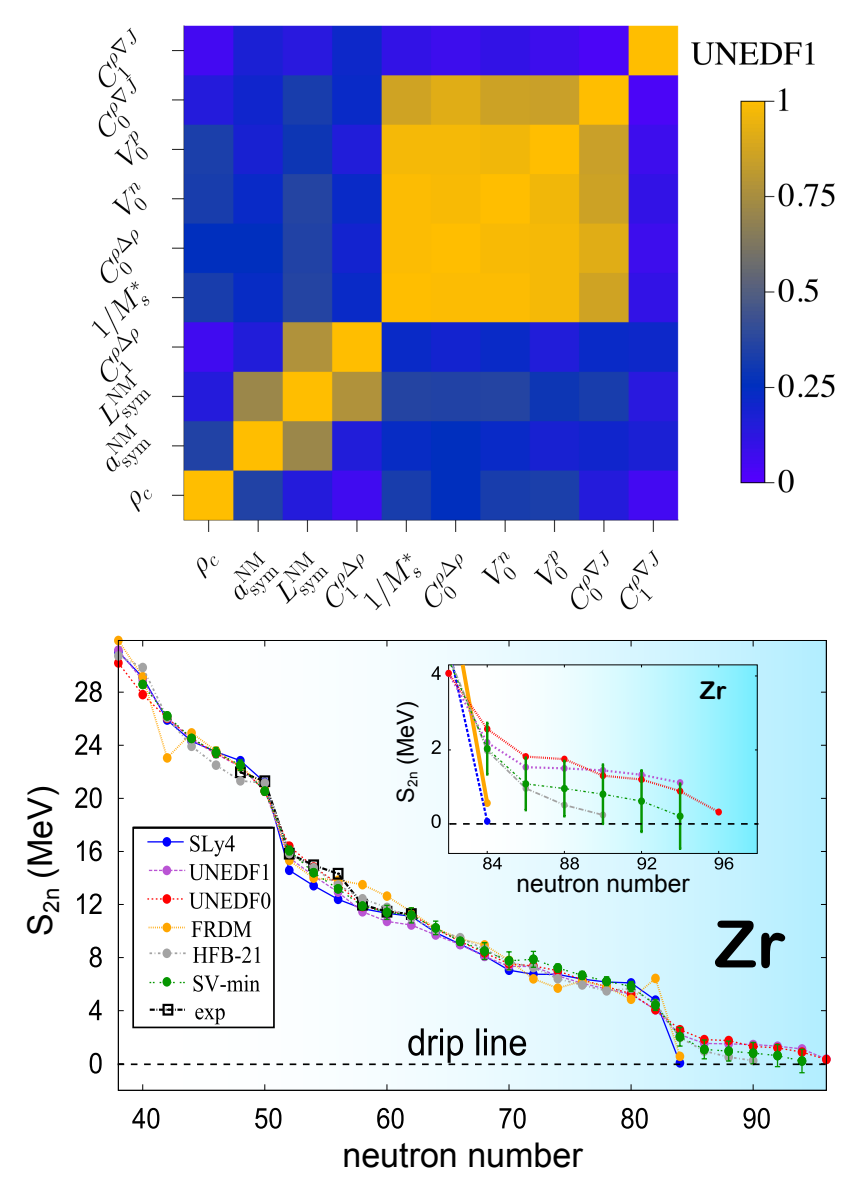

Figure 20: Top: UNEDF1 correlation matrix. Presented are the absolute values of the correlation coefficients between the parameters characterizing the energy density (1). Bottom: Theoretical extrapolations toward drip lines for the two-neutron separation energies $S_{2 n}$ for the isotopic chain of even-even $\mathrm{Zr}$ isotopes using different EDFs (SLy4, SV-min, UNEDF0, UNEDF1) 76 and FRDM 163 and HFB-21 164 mass models. Detailed predictions around $S_{2 n}=0$ are illustrated in the inset. The bars on the SV-min results indicate statistical errors due to uncertainty in the coupling constants of the functional.

Intrepid is an IBM Blue Gene/ $\mathrm{P}$ with 163,840 processing cores.

Figure 21 shows the UNEDF utilization of these computing resources over the years 2008-2013 provided through INCITE. The figure highlights the increasing demand for computing time in low-energy nuclear physics research. The combined 2008 INCITE utilization across Jaguar and Intrepid was nearly 20 million core-hours and by 2012 had increased fourfold. This growth illustrates the increasing application of high-performance computing in nuclear theory enabled by the physics/computer science/applied mathematics collaborations fostered by UNEDF.

For the 2013 calendar year, members of the SciDAC-3 NUCLEI project 172 were granted the sixth largest allocation of the 61 INCITE projects awarded, with a total allocation of 155 million core-hours across three leadershipclass computing resources, Titan, Mira, and Intrepid. Ti- 


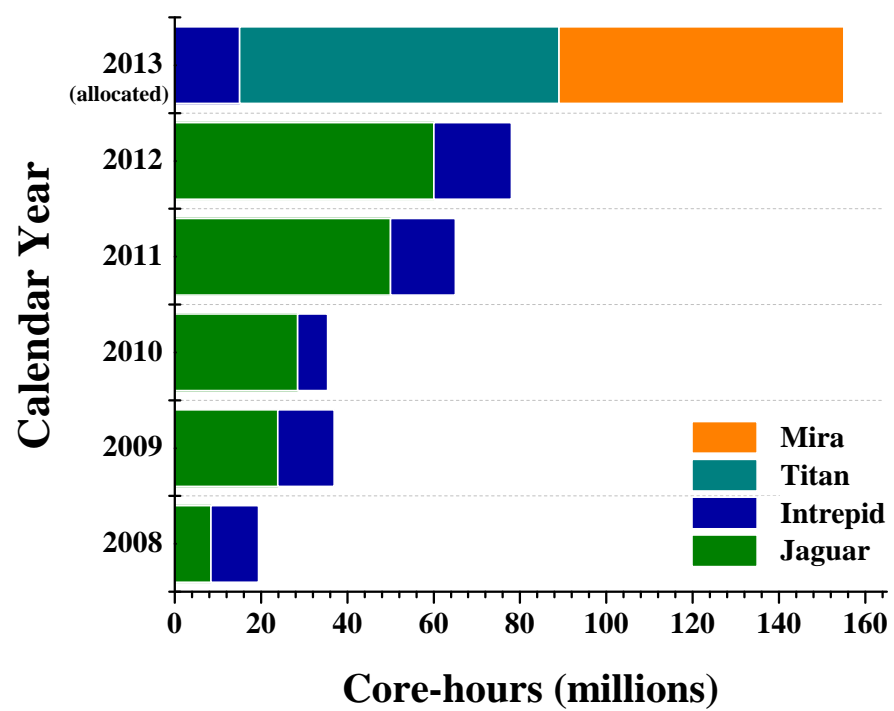

Figure 21: UNEDF allocation and utilization (in millions of corehours) of leadership-class computing resources from 2008 to 2013.

tan is a Cray XK7, a hybrid CPU-GPU system with 299,008 CPU cores and 261,632 GPU streaming multiprocessors, and Mira is an IBM Blue Gene/Q with 786,432 processing cores. The substantial changes to computing systems at both Argonne and Oak Ridge, indicative of future trends in high-performance computing, create new computational challenges but also new possibilities to achieve larger and more accurate calculations. Through the close collaborations enabled through UNEDF, and now NUCLEI, members are working to continuously scale codes to increase physics capabilities and improve performance for efficient utilization of these leadership-class resources.

\section{Conclusions}

The examples presented here illustrate the multifaceted outcomes of the UNEDF project, both in terms of landmark calculations of nuclear structure and reactions and in terms of how nuclear theory is done. The project was very productive, as can be assessed by going to the project's website, http://unedf.org, which documents the concrete deliverables of UNEDF: publications, highlights, reports, conference presentations, and computer codes. UNEDF also placed great importance on recruiting the next generation of scientists. Annually it provided training to 30 young researchers. The UNEDF experience has been a springboard for advancement, with many UNEDF postdocs obtaining permanent positions at U.S. universities, national laboratories, and overseas institutions.

By fostering broad new collaborative efforts between physicists, mathematicians, and computer scientists, the SciDAC-2 UNEDF project showed how to tackle scientific, algorithmic, and computational challenges in the era of extreme-scale scientific computing. This effort continues with the SciDAC-3 NUCLEI project [172, which builds

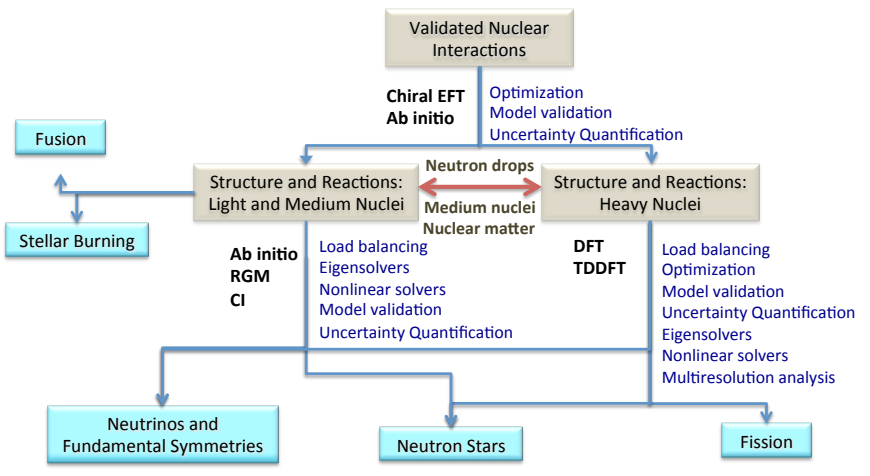

Figure 22: Physics and computing in NUCLEI. The major areas of research are marked, together with connections between them and theoretical and computational tools. For more details, see [172.

on the successful strategies of UNEDF. Figure 22 shows the key elements of NUCLEI.

\section{Acknowledgments}

Support for the UNEDF and NUCLEI collaborations was provided through the SciDAC program funded by the U.S. Dept. of Energy (DOE), Office of Science, Advanced Scientific Computing Research and Nuclear Physics programs. This work was also supported by DOE Contract Nos. DE-FG02-96ER40963 (Univ. Tenn.), DE-AC5207NA27344 (LLNL), DE-AC02-05CH11231 (LBNL), DEAC05-00OR22725 (ORNL), DE-AC02-06CH11357 and DE-FC02-07ER41457 (ANL), DE-FC02-09ER41584 (Central Michigan Univ.), DE-FC02-09ER41582 (Iowa State Univ.), DE-FG02-87ER40371 (Iowa State Univ.), and DEFC02-09ER41586 (Ohio State Univ.). This research used the computational resources of the Oak Ridge Leadership Computing Facility (OLCF) at ORNL and Argonne Leadership Computing Facility (ALCF) at ANL provided through the INCITE program. Computational resources were also provided by the National Institute for Computational Sciences (NICS) at ORNL, the Laboratory Computing Resource Center (LCRC) at ANL, and the National Energy Research Scientific Computing Center (NERSC) at LBNL.

\section{References}

[1] G. F. Bertsch, D. J. Dean, W. Nazarewicz, SciDAC Rev. 6 (2007) 42.

[2] R. Furnstahl, Nucl. Phys. News 21 (2011) 24.

[3] J. Carlson, Phys. Rev. C 36 (1987) 2026.

[4] S. C. Pieper, R. B. Wiringa, Annu. Rev. Nucl. Part. Sci. 51 (2001) 53.

[5] E. Lusk, S. C. Pieper, R. Butler, SciDAC Rev. 17 (2010) 30.

[6] K. M. Nollett, S. C. Pieper, R. B. Wiringa, J. Carlson, G. M. Hale, Phys. Rev. Lett. 99 (2007) 022502.

[7] B. S. Pudliner, V. R. Pandharipande, J. Carlson, S. C. Pieper, R. B. Wiringa, Phys. Rev. C 56 (1997) 1720.

[8] R. B. Wiringa, S. C. Pieper, J. Carlson, V. R. Pandharipande, Phys. Rev. C 62 (2000) 014001. 
[9] S. C. Pieper, R. B. Wiringa, J. Carlson, Phys. Rev. C 70 (2004) 054325 .

[10] E. Epelbaum, H. Krebs, D. Lee, U.-G. Meißner, Phys. Rev. Lett. 106 (2011) 192501.

[11] E. Epelbaum, H. Krebs, T. A. Lahde, D. Lee, U.-G. Meißner, Phys. Rev. Lett. 109 (2012) 252501.

[12] R. B. Wiringa, R. Schiavilla, S. C. Pieper, J. Carlson, Phys. Rev. C 78 (2008) 021001.

[13] M. Pervin, S. C. Pieper, R. B. Wiringa, Phys. Rev. C 76 (2007) 064319

[14] S. Aroua, P. Navrátil, L. Zamick, M. S. Fayache, B. R. Barrett, J. P. Vary, K. Heyde, Nucl. Phys. A 720 (2011) 71.

[15] P. Maris, J. P. Vary, P. Navratil, W. E. Ormand, H. Nam, D. J. Dean, Phys. Rev. Lett. 106 (2011) 202502.

[16] P. Sternberg, E. G. Ng, C. Yang, P. Maris, J. P. Vary, M. Sosonkina, H. V. Le, Accelerating configuration interaction calculations for nuclear structure, in: Proc. 2008 ACM/IEEE Conf. Supercomputing, 2008.

[17] J. P. Vary, P. Maris, E. Ng, C. Yang, M. Sosonkina, J. Phys. Conf. Ser. 180 (2009) 012083.

[18] P. Maris, M. Sosonkina, J. P. Vary, E. Ng, C. Yang, Proc. Comp. Sci. 1 (2010) 97.

[19] H. Aktulga, C. Yang, E. Ng, P. Maris, J. Vary, LCNS 7484 (2012) 830

[20] J. W. Holt, G. E. Brown, T. T. S. Kuo, J. D. Holt, R. Machleidt, Phys. Rev. Lett. 100 (2008) 062501.

[21] B. R. Barrett, P. Navrátil, J. P. Vary, Prog. Part. Nucl. Phys. 69 (2013) 131.

[22] P. Maris, H. M. Aktulga, M. A. Caprio, U. Catalyurek, E. G. Ng, et al., J. Phys.: Conf. Ser. 403 (2012) 012019.

[23] P. Maris, A. Shirokov, J. Vary, Phys. Rev. C C81 (2010) 021301 .

[24] P. Maris, J. P. Vary, S. Gandolfi, J. Carlson, S. C. Pieper, arXiv:1302.2089.

[25] S. Quaglioni, P. Navrátil, Phys. Rev. Lett. 101 (2008) 092501.

[26] S. Quaglioni, P. Navrátil, Phys. Rev. C 79 (2009) 044606.

[27] S. Baroni, P. Navrátil, S. Quaglioni, Phys. Rev. Lett. 110 (2013) 022505.

[28] P. Navrátil, S. Quaglioni, Phys. Rev. Lett. 108 (2012) 042503.

[29] P. Navrátil, R. Roth, S. Quaglioni, Phys. Lett. B 704 (2011) 379

[30] F. Coester, Nucl. Phys. 7 (1958) 421.

[31] F. Coester, H. Kümmel, Nucl. Phys. 17 (1960) 477.

[32] H. Kümmel, K. H. Lührmann, J. G. Zabolitzky, Phys. Rep. 36 (1978) 1.

[33] R. J. Bartlett, M. Musiał, Rev. Mod. Phys. 79 (2007) 291.

[34] D. J. Dean, M. Hjorth-Jensen, Phys. Rev. C 69 (2004) 054320.

[35] G. Hagen, T. Papenbrock, D. J. Dean, M. Hjorth-Jensen, Phys. Rev. C 82 (2010) 034330.

[36] D. R. Entem, R. Machleidt, Phys. Rev. C 68 (2003) 041001.

[37] G. Hagen, T. Papenbrock, D. J. Dean, M. Hjorth-Jensen, Phys. Rev. Lett. 101 (2008) 092502.

[38] G. Hagen, D. J. Dean, M. Hjorth-Jensen, T. Papenbrock, Phys. Lett. B 656 (2007) 169

[39] G. Hagen, T. Papenbrock, M. Hjorth-Jensen, Phys. Rev. Lett. 104 (2010) 182501.

[40] G. Hagen, T. Papenbrock, D. J. Dean, Phys. Rev. Lett. 103 (2009) 062503.

[41] G. R. Jansen, M. Hjorth-Jensen, G. Hagen, T. Papenbrock, Phys. Rev. C 83 (2011) 054306.

[42] J. W. Holt, N. Kaiser, W. Weise, Phys. Rev. C 79 (2009) 054331 .

[43] K. Hebeler, A. Schwenk, Phys. Rev. C 82 (2010) 014314.

[44] G. Hagen, M. Hjorth-Jensen, G. R. Jansen, R. Machleidt, T. Papenbrock, Phys. Rev. Lett. 108 (2012) 242501

[45] R. J. Furnstahl, G. Hagen, T. Papenbrock, Phys. Rev. C 86 (2012) 031301

[46] G. Hagen, T. Papenbrock, D. J. Dean, M. Hjorth-Jensen, B. V. Asokan, Phys. Rev. C 80 (2009) 021306.

[47] G. Hagen, H. A. Nam, Prog. Theor. Phys., Suppl. 196 (2012) 102 .
[48] G. Hagen, D. J. Dean, M. Hjorth-Jensen, T. Papenbrock, A. Schwenk, Phys. Rev. C 76 (2007) 044305.

[49] G. Hagen, M. Hjorth-Jensen, G. R. Jansen, R. Machleidt, T. Papenbrock, Phys. Rev. Lett. 109 (2012) 032502

[50] J. I. Prisciandaro, P. F. Mantica, B. A. Brown, D. W. Anthony, M. W. Cooper, A. Garcia, D. E. Groh, A. Komives, W. Kumarasiri, P. A. Lofy, A. M. Oros-Peusquens, S. L. Tabor, M. Wiedeking, Phys. Lett. B 510 (2001) 17.

[51] R. V. F. Janssens, B. Fornal, P. F. Mantica, B. A. Brown, R. Broda, P. Bhattacharyya, M. P. Carpenter, M. Cinausero, P. J. Daly, A. D. Davies, T. Glasmacher, Z. W. Grabowski, D. E. Groh, M. Honma, F. G. Kondev, W. Królas, T. Lauritsen, S. N. Liddick, S. Lunardi, N. Marginean, T. Mizusaki, D. J. Morrissey, A. C. Morton, W. F. Mueller, T. Otsuka, T. Pawlat, D. Seweryniak, H. Schatz, A. Stolz, S. L. Tabor, C. A. Ur, G. Viesti, I. Wiedenhaver, J. Wrzesinski, Phys. Lett. B 546 (2002) 55 .

[52] M. Honma, T. Otsuka, B. A. Brown, T. Mizusaki, Phys. Rev. C 65 (2002) 061301

[53] S. N. Liddick, P. F. Mantica, R. V. F. Janssens, R. Broda, B. A. Brown, M. P. Carpenter, B. Fornal, M. Honma, T. Mizusaki, A. C. Morton, W. F. Mueller, T. Otsuka, J. Pavan, A. Stolz, S. L. Tabor, B. E. Tomlin, M. Wiedeking, Phys. Rev. Lett. 92 (2004) 072502.

[54] D.-C. Dinca, R. V. F. Janssens, A. Gade, D. Bazin, R. Broda, B. A. Brown, C. M. Campbell, M. P. Carpenter, P. Chowdhury, J. M. Cook, A. N. Deacon, B. Fornal, S. J. Freeman, T. Glasmacher, M. Honma, F. G. Kondev, J.-L. Lecouey, S. N. Liddick, P. F. Mantica, W. F. Mueller, H. Olliver, T. Otsuka, J. R. Terry, B. A. Tomlin, K. Yoneda, Phys. Rev. C 71 (2005) 041302.

[55] U. van Kolck, Phys. Rev. C 49 (1994) 2932.

[56] E. Epelbaum, H.-W. Hammer, U.-G. Meißner, Rev. Mod. Phys. 81 (2009) 1773.

[57] R. Machleidt, D. Entem, Phys. Rep. 503 (2011) 1.

[58] J. D. Holt, T. Otsuka, A. Schwenk, T. Suzuki, J. Phys. G 39 (2012) 085111

[59] D. Steppenbeck, private communication (2012).

[60] C. W. Johnson, W. E. Ormand, P. G. Krastev, arXiv: 1303.0905

[61] E. Caurier, F. Nowacki, Acta Phys. Pol. B 30 (1999) 705

[62] E. Caurier, G. Martínez-Pinedo, F. Nowacki, A. Poves, J. Retamosa, A. P. Zuker, Phys. Rev. C 59 (1999) 2033.

[63] H. Aktulga, C. Yang, E. Ng, P. Maris, J. Vary, Large-scale parallel null space calculation for nuclear configuration interaction problem, in: Proc. Intern. Conf. on High Performance Computing and Simulation, 2011.

[64] H. Aktulga, C. Yang, U. Catalyurek, P. Maris, J. Vary, E. Ng, On reducing $\mathrm{I} / \mathrm{O}$ overheads in large-scale invariant subspace projections, in: Proc. 2011 Workshop on Algorithms and Programming Tools for Next-Generation High-Performance Scientific Software, 2011, p. 305.

[65] NuShellX, see http://www.garsington.eclipse.co.uk

[66] M. Bender, P.-H. Heenen, P.-G. Reinhard, Rev. Mod. Phys. 75 (2003) 121.

[67] Y. M. Engel, D. M. Brink, K. Goeke, S. Krieger, D. Vautherin, Nucl. Phys. A 249 (1975) 215.

[68] S. G. Rohoziński, J. Dobaczewski, W. Nazarewicz, Phys. Rev. C 81 (2010) 014313.

[69] J. Dobaczewski, H. Flocard, J. Treiner, Nucl. Phys. A 422 (1984) 103.

[70] J. C. Pei, A. T. Kruppa, W. Nazarewicz, Phys. Rev. C 84 (2011) 024311

[71] M. Kortelainen, T. Lesinski, J. Moré, W. Nazarewicz, J. Sarich, N. Schunck, M. V. Stoitsov, S. Wild, Phys. Rev. C 82 (2010) 024313.

[72] M. Kortelainen, J. McDonnell, W. Nazarewicz, P.-G. Reinhard, J. Sarich, N. Schunck, M. Stoitsov, S. Wild, Phys. Rev. C 85 (2012) 024304

[73] M. V. Stoitsov, J. Dobaczewski, W. Nazarewicz, S. Pittel, D. J. Dean, Phys. Rev. C 68 (2003) 054312. 
[74] M. Stoitsov, W. Nazarewicz, N. Schunck, Int. J. Mod. Phys. 18 (2009) 816.

[75] J. Erler, N. Birge, M. Kortelainen, W. Nazarewicz, E. Olsen, A. M. Perhac, M. Stoitsov, J. Phys.: Conf. Ser. 402 (2012) 012030 .

[76] J. Erler, N. Birge, M. Kortelainen, W. Nazarewicz, E. Olsen, A. M. Perhac, M. Stoitsov, Nature 486 (2012) 509.

[77] M. Stoitsov, J. Dobaczewski, W. Nazarewicz, P. Ring, Comp. Phys. Comm. 167 (2005) 43.

[78] J. Dobaczewski, J. Dudek, Comp. Phys. Comm. 102 (1997) 183

[79] M. Stoitsov, N. Schunck, M. Kortelainen, N. Michel, H. Nam, E. Olsen, J. Sarich, S. Wild, Comp. Phys. Comm. 184 (2013) 1592.

[80] N. Schunck, J. Dobaczewski, J. McDonnell, W. Satuła, J. Sheikh, A. Staszczak, M. Stoitsov, P. Toivanen, Comp. Phys. Comm. 183 (2012) 166.

[81] A. Staszczak, A. Baran, J. Dobaczewski, W. Nazarewicz, Phys. Rev. C 80 (2009) 014309.

[82] Y. Shi, J. Dobaczewski, S. Frauendorf, W. Nazarewicz, J. Pei, F. Xu, N. Nikolov, Phys. Rev. Lett. 108 (2012) 092501.

[83] N. Schunck, J. Dobaczewski, J. McDonnell, J. Moré, W. Nazarewicz, J. Sarich, M. V. Stoitsov, Phys. Rev. C 81 (2010) 024316 .

[84] A. Staszczak, M. Stoitsov, A. Baran, W. Nazarewicz, Euro. Phys. J. A 46 (2010) 85.

[85] A. Baran, A. Bulgac, M. Forbes, G. Hagen, W. Nazarewicz, N. Schunck, M. V. Stoitsov, Phys. Rev. C 78 (2008) 014318.

[86] J. C. Pei, M. V. Stoitsov, G. I. Fann, W. Nazarewicz, N. Schunck, F. R. Xu, Phys. Rev. C 78 (2008) 064306.

[87] J. Pei, W. Nazarewicz, J. Sheikh, A. Kerman, Phys. Rev. Lett. 102 (2009) 192501.

[88] J. C. Pei, J. Dukelsky, W. Nazarewicz, Phys. Rev. A 82 (2010) 021603 .

[89] R. Parrish, E. Hoenstein, N. Schunck, C. Sherril, T. Martinez, arXiv:1301.5064

[90] B. Alpert, G. Beylkin, D. Gines, L. Vozovoi, J. Computat. Phys. 182 (2002) 149

[91] B. Alpert, G. Beylkin, R. Coifman, V. Rokhlin, SIAM J. Sci. Comput. 14 (1993) 159.

[92] M. Brewster, G. Fann, Z. Yang, J. Math. Chem. 22 (1997) 117.

[93] G. Fann, G. Beylkin, R. Harrison, K. Jordan, IBM J. Res. Dev. 48 (2004) 161

[94] T. Yanai, G. I. Fann, Z. Gan, R. J. Harrison, G. Beylkin, J. Chem. Phys. 121 (2004) 6680

[95] G. Beylkin, R. Cramer, G. Fann, R. J. Harrison, J. Appl. Comp. Harmonic Anal. 23 (2007) 235.

[96] N. Vence, R. Harrison, P. Krstić, Phys. Rev. A 85 (2012) 033403

[97] J. Jia, R. J. Harrison, G. Fann, submitted.

[98] K. Bennaceur, J. Dobaczewski, Comp. Phys. Comm. 168 (2005) 96.

[99] G. I. Fann, J. Pei, R. J. Harrison, J. Jia, J. Hill, M. Ou, W. Nazarewicz, W. A. Shelton, N. Schunck, J. Phys.: Conf. Ser. 180

[100] N. Nikolov, N. Schunck, W. Nazarewicz, M. Bender, J. Pei, Phys. Rev. C 83 (2011) 034305.

[101] T. Munson, J. Sarich, S. M. Wild, S. Benson, L. Curfman McInnes, TAO 2.0 users manual, Technical Memorandum ANL/MCS-TM-322, Argonne National Laboratory, Argonne, Illinois, see http://www.mcs.anl.gov/tao (2012).

[102] S. Gandolfi, J. Carlson, S. Reddy, Phys. Rev. C 85 (2012) 032801

[103] M. B. Tsang, J. R. Stone, F. Camera, P. Danielewicz, S. Gandolfi, K. Hebeler, C. J. Horowitz, J. Lee, W. G. Lynch, Z. Kohley, R. Lemmon, P. Möller, T. Murakami, S. Riordan, X. RocaMaza, F. Sammarruca, A. W. Steiner, I. Vidaña, S. J. Yennello, Phys. Rev. C 86 (2012) 015803.

[104] S. Gandolfi, A. Y. Illarionov, S. Fantoni, J. Miller, F. Pederiva, K. Schmidt, MNRAS 404 (2010) L35.

[105] S. Gandolfi, A. Y. Illarionov, K. E. Schmidt, F. Pederiva
S. Fantoni, Phys. Rev. C 79 (2009) 054005

[106] S.-Y. Chang, J. Morales, V. R. Pandharipande, D. G. Ravenhall, J. Carlson, S. C. Pieper, R. B. Wiringa, K. E. Schmidt, Nucl. Phys. A 746 (2004) 215

[107] S. Gandolfi, F. Pederiva, S. Fantoni, K. E. Schmidt, Phys. Rev. C 73 (2006) 044304.

[108] S. Gandolfi, F. Pederiva, S. A Beccara, Euro. Phys. J. A 35 (2008) 207.

[109] S. Gandolfi, J. Carlson, S. C. Pieper, Phys. Rev. Lett. 106 (2011) 012501

[110] A. W. Steiner, S. Gandolfi, Phys. Rev. Lett. 108 (2012) 081102.

[111] M. Stoitsov, M. Kortelainen, S. K. Bogner, T. Duguet, R. J. Furnstahl, B. Gebremariam, N. Schunck, Phys. Rev. C 82 (2010) 054307.

[112] S. K. Bogner, R. J. Furnstahl, H. Hergert, M. Kortelainen, P. Maris, M. Stoitsov, J. P. Vary, Phys. Rev. C 84 (2011) 044306

[113] J. E. Drut, R. J. Furnstahl, L. Platter, Prog. Part. Nucl. Phys. 64 (2010) 120

[114] E. D. Jurgenson, P. Navratil, R. J. Furnstahl, Phys. Rev. Lett. 103 (2009) 082501.

[115] S. K. Bogner, R. J. Furnstahl, A. Schwenk, Prog. Part. Nucl. Phys. 65 (2010) 94

[116] E. D. Jurgenson, P. Navrátil, R. J. Furnstahl, Phys. Rev. C 83 (2011) 034301

[117] R. Roth, J. Langhammer, A. Calci, S. Binder, P. Navrátil, Phys. Rev. Lett. 107 (2011) 072501.

[118] R. Roth, S. Binder, K. Vobig, A. Calci, J. Langhammer, P. Navrátil, Phys. Rev. Lett. 109 (2012) 052501.

[119] K. Hebeler, S. Bogner, R. Furnstahl, A. Nogga, A. Schwenk, Phys. Rev. C 83 (2011) 031301.

[120] J. W. Negele, D. Vautherin, Phys. Rev. C 5 (1972) 1472.

[121] S. K. Bogner, R. J. Furnstahl, L. Platter, Euro. Phys. J. A 39 (2009) 219.

[122] B. Gebremariam, T. Duguet, S. K. Bogner, Phys. Rev. C 82 (2010) 014305

[123] B. Carlsson, J. Dobaczewski, Phys. Rev. Lett. 105 (2010) 122501 .

[124] J. Dobaczewski, B. Carlsson, M. Kortelainen, J. Phys. G 37 (2010) 075106 .

[125] J. Terasaki, J. Engel, M. Bender, J. Dobaczewski, W. Nazarewicz, M. Stoitsov, Phys. Rev. C 71 (2005) 034310.

[126] J. Terasaki, J. Engel, Phys. Rev. C 82 (2010) 034326.

[127] K. Yoshida, Phys. Rev. C 80 (2009) 044324.

[128] S. Ebata, T. Nakatsukasa, T. Inakura, K. Yoshida, Y. Hashimoto, K. Yabana, Phys. Rev. C 82 (2010) 034306.

[129] D. P. Arteaga, E. Khan, P. Ring, Phys. Rev. C 79 (2009) 034311

[130] S. Peru, G. Gosselin, M. Martini, M. Dupuis, S. Hilaire, J.-C. Devaux, Phys. Rev. C 83 (2011) 014314.

[131] C. Losa, A. Pastore, T. Dossing, E. Vigezzi, R. Broglia, Phys. Rev. C 81 (2010) 064307.

[132] P. Ring, P. Schuck, The Nuclear Many-body Problem, Springer-Verlag, Berlin, 1980.

[133] A. Blazkiewicz, V. E. Oberacker, A. S. Umar, M. Stoitsov, Phys. Rev. C 71 (2005) 054321.

134] J. Terasaki, J. Engel, Phys. Rev. C 84 (2011) 014332

[135] G. P. A. Nobre, F. S. Dietrich, J. E. Escher, I. J. Thompson, M. Dupuis, J. Terasaki, J. Engel, Phys. Rev. Lett. 105 (2010) 202502

[136] G. P. A. Nobre, F. S. Dietrich, J. E. Escher, I. J. Thompson, M. Dupuis, J. Terasaki, J. Engel, Phys. Rev. C 84 (2011) 064609

[137] G. P. A. Nobre, I. J. Thompson, J. E. Escher, F. S. Dietrich, J. Phys.: Conf. Ser. 312 (2011) 082033.

[138] A. J. Koning, J. P. Delaroche, Nucl. Phys. A 713 (2003) 231.

[139] J. J. H. Menet, E. E. Gross, J. J. Malanify, A. Zucker, Phys. Rev. C 4 (1971) 1114

140] P. Avogadro, T. Nakatsukasa, Phys. Rev. C 84 (2011) 014314.

[141] P. Avogadro, T. Nakatsukasa, Phys. Rev. C 87 (2013) 014331. 
[142] M. Stoitsov, M. Kortelainen, T. Nakatsukasa, C. Losa, W. Nazarewicz, Phys. Rev. C 84 (2011) 041305(R).

[143] J. Toivanen, B. G. Carlsson, J. Dobaczewski, K. Mizuyama, R. R. Rodríguez-Guzmán, P. Toivanen, P. Veselý, Phys. Rev. C 81 (2010) 034312.

[144] B. G. Carlsson, J. Toivanen, A. Pastore, Phys. Rev. C 86 (2012) 014307.

[145] A. Bulgac, Y. Yu, Phys. Rev. Lett. 88 (2002) 042504.

[146] A. Bulgac, Phys. Rev. C 65 (2002) 051305(R).

[147] A. Bulgac, Phys. Rev. A 76 (2007) 040502.

[148] A. Bulgac, M. M. Forbes, Phys. Rev. Lett. 101 (2008) 215301.

[149] A. Bulgac, S. Yoon, Phys. Rev. Lett. 102 (2009) 085302.

[150] A. Bulgac, M. M. Forbes, P. Magierski, The Unitary Fermi Gas: From Monte Carlo to Density Functionals, Vol. 836, Springer-Verlag, Berlin, 2012, Ch. 9, p. 305.

[151] A. Bulgac, Y.-L. Luo, P. Magierski, K. J. Roche, Y. Yu, Science 332 (2011) 1288.

[152] A. Bulgac, Y.-L. Luo, K. J. Roche, Phys. Rev. Lett. 108 (2012) 150401.

[153] I. Stetcu, A. Bulgac, P. Magierski, K. J. Roche, Phys. Rev. C 84 (2011) 051309(R).

[154] A. Bulgac, arXiv:1301.0357.

[155] A. Bulgac, K. J. Roche, J. Phys.: Conf. Ser. 125 (2008) 012064 .

[156] A. Bulgac, M. M. Forbes, arXiv:1301.7354.

[157] W. Hauser, H. Feshbach, Phys. Rev. 87 (1952) 366.

[158] M. Scott, M. Horoi, Euro. Phys. Lett. 91 (2010) 52001.

[159] M. Horoi, V. Zelevinsky, Phys. Rev. Lett. 98 (2007) 262503.

[160] R. Sen'kov, M. Horoi, Phys. Rev. C 82 (2010) 024304.

[161] R. Sen'kov, M. Horoi, V. Zelevinsky, Phys. Lett. B 702 (2011) 413.

[162] R. Sen'kov, M. Horoi, V. Zelevinsky, Comp. Phys. Comm. 184 (2013) 215

[163] P. Möller, J. R. Nix, W. J. Myres, Swiatecki, At. Data Nucl. Data Tables 59 (1995) 185.

[164] S. Goriely, N. Chamel, J. M. Pearson, Phys. Rev. C 82 (2010) 035804 .

[165] G. F. Bertsch, B. Sabbey, M. Uusnäkki, Phys. Rev. C 71 (2005) 054311.

[166] J. Toivanen, J. Dobaczewski, M. Kortelainen, K. Mizuyama, Phys. Rev. C 78 (2008) 034306.

[167] P. Klüpfel, P.-G. Reinhard, T. J. Bürvenich, J. A. Maruhn, Phys. Rev. C 79 (2009) 034310.

[168] P.-G. Reinhard, W. Nazarewicz, Phys. Rev. C 81 (2010) 051303.

[169] J. J. Moré, S. M. Wild, SIAM J. Sci. Comput. 33 (2011) 1292.

[170] J. J. Moré, S. M. Wild, ACM Trans. Math. Soft. 38 (2012) $19: 1$.

[171] U.S. Department of Energy, DOE Leadership Computing, see www.doeleadershipcomputing.org

[172] NUCLEI: Nuclear computational low-energy initiative, see http://computingnuclei.org 